\title{
Modification of conductivity due to acceleration of the ionospheric medium
}

\author{
V. V. Denisenko ${ }^{1,2}$, H. K. Biernat ${ }^{3}$, A. V. Mezentsev $^{1}$, V. A. Shaidurov ${ }^{1}$, and S. S. Zamay ${ }^{1}$ \\ ${ }^{1}$ Institute of Computational Modelling, Russian Academy of Sciences, Siberian Branch, Krasnoyarsk, 660036, Russia \\ ${ }^{2}$ Siberian Federal University, Krasnoyarsk, 660041, Russia \\ ${ }^{3}$ Space Research Institute, Austrian Academy of Sciences, Schmiedlstrasse 6, 8042 Graz, Austria
}

Received: 23 February 2006 - Revised: 16 April 2008 - Accepted: 16 May 2008 - Published: 31 July 2008

\begin{abstract}
A quantitative division of the ionosphere into dynamo and motor regions is performed on the base of empirical models of space distributions of ionospheric parameters. Pedersen and Hall conductivities are modified to represent an impact of acceleration of the medium because of Ampére's force. It is shown that the currents in the $F_{2}$ layer are greatly reduced for processes of a few hours duration. This reduction is in particular important for the night-side low-latitude ionosphere. The International Reference Ionosphere model is used to analyze the effect quantitatively. This model gives a second high conducting layer in the night-side low-latitude ionosphere that reduces the electric field and equatorial electrojets, but intensifies night-side currents during the shortterm events. These currents occupy regions which are much wider than those of equatorial electrojets.

It is demonstrated that the parameter $\sigma_{d}=\sigma_{P}+\sigma_{H} \Sigma_{H} / \Sigma_{P}$ that involves the integral Pedersen and Hall conductances $\Sigma_{P}, \Sigma_{H}$ ought to be used instead of the local Cowling conductivity $\sigma_{C}$ in calculations of the electric current density in the equatorial ionosphere. We may note that Gurevich et al. (1976) derived a parameter similar to $\sigma_{d}$ for more general conditions as those which we discuss in this paper; a more detailed description of this point is given in Sect. 6. Both, $\sigma_{d}$ and $\sigma_{C}$, appear when a magnetic field line is near a nonconducting domain which means zero current through the boundary of this domain. The main difference between $\sigma_{d}$ and $\sigma_{C}$ is that $\sigma_{d}$ definition includes the possibility for the electric current to flow along a magnetic field line in order to close all currents which go to this line from neighboring ones. The local Cowling conductivity $\sigma_{C}$ corresponds to the current closure at each point of a magnetic field line. It is adequate only for a magnetic field line with constant local conductivity at the whole line when field-aligned currents do not exist because of symmetry, but $\sigma_{C}=\sigma_{d}$ in this case. So,
\end{abstract}

Correspondence to: $\mathrm{H}$. K. Biernat

(helfried.biernat@oeaw.ac.at) there is no reason to use the local Cowling conductivity while the Cowling conductance $\Sigma_{C}=\Sigma_{P}+\Sigma_{H}^{2} / \Sigma_{P}$ is a useful and well defined parameter.

Keywords. Ionosphere (Electric fields and currents; Equatorial ionosphere; Modeling and forecasting)

\section{Introduction}

The ionosphere is usually considered as a conductor with a given conductivity distribution when global electric fields and currents are simulated. Considering the high conductivity in the direction of the magnetic field, a two-dimensional approach is appropriate. The magnetic field lines are equipotentials and the ionospheric conductor may be represented by Pedersen and Hall conductances which are equal to integrals along magnetic field lines of the corresponding local conductivities $\sigma_{P}, \sigma_{H}$ (Gurevich et al., 1976).

If the conductor is moving, then Ohm's law is valid in the moving frame of reference. An additional term appears in the laboratory frame of reference that is proportional to the velocity. This kind of electric field generator is subject of the dynamo theory. The motion of the medium is mainly defined by neutral winds and it is slightly disturbed by the Ampére force in the E-region of the ionosphere since the density is large there. So, this conductor moves in the magnetic field and works as a magnetohydrodynamic generator. In contrast to the E-region, the medium in the F-region is guided by the Ampére force that corresponds to a division of the ionosphere into dynamo and motor regions (Hargreaves, 1979).

The purpose of the paper is to analyze the motion of the conducting medium, whose appearance is due to the electric and magnetic fields, and to estimate how the electric current is changed due to its influence. This gives a quantitative description of the ionospheric region in that the dynamo is changed by the motor.

Published by Copernicus Publications on behalf of the European Geosciences Union. 
It is always difficult to estimate a common result of many factors when they exist altogether. So, we use a simplified way to analyze the factors separately. Such an approach may be insufficient for nonlinear systems when few factors are strong, but it is useful as a step in research. A pressure gradient is taken into account in the frame of a local model (Maeda, 1977). A complicated model like this of Namgaladze et al. (2000) is necessary to analyze all forces which define the motion in the ionosphere.

The motion of the medium may be approximately taken into account as a modification of the conductivity. The concept of defining some effective conductivity which represents a moving conducting medium is not new. It is done by Akasofu and Chapman (1972) under the assumption of a steadystate motion. It takes different time to accelerate electrons, ions, and neutrals up to a steady-state motion. The conductivity dependence of the electric field on the frequency is analyzed by Vanyan et al. (1973) in the case when neutrals are almost at rest. The model (Akasofu and Dewitt, 1965) represents a modification of the Pedersen conductivity for an electric field which is harmonic in time. Our approach differs mainly in that an unsteady process is regarded as a relaxation to a new steady state after a moment when the electric field is changed. We add an analysis of integrated conductivities which is important for models of large scale electric fields.

In this paper, we analyze only local effects. A global problem aught be solved with this modified conductivity to find a more realistic electric field. Such a problem is described in Sect. 5.

\section{The conductor motion}

Let us consider a homogeneous conductor which moves in magnetic $\mathbf{B}$ and electric $\mathbf{E}$ fields. Let us split the vectors $\mathbf{E}$ into field-aligned components $E_{\|}$parallel to the magnetic field and the normal components $\mathbf{E}_{\perp}$. The $\mathrm{Ohm}$ law is valid in the rest frame of reference,

$j_{\|}=\sigma_{\|} E_{\|}$,

$\mathbf{j}_{\perp}=\sigma_{P} \mathbf{E}_{\perp}^{\prime}-\sigma_{H}\left[\mathbf{E}_{\perp}^{\prime} \times \mathbf{B}\right] / B$,

$\mathbf{E}_{\perp}^{\prime}=\mathbf{E}_{\perp}+\left[\mathbf{u}_{\perp} \times \mathbf{B}\right]$,

where $\mathbf{j}$ is the current density, quantities $\sigma_{P}, \sigma_{H}, \sigma_{\|}$are the Pedersen, Hall, and field-aligned conductivities and $\mathbf{u}$ is the conductor velocity. We use SI units. In the case of our interest, the electric field and velocity are normal to the magnetic field.

Since we analyze separately the motion of the conducting medium, whose appearance is due to the electric and magnetic fields, no force but the Ampére one needs be taken into account in the equation of motion

$\rho \frac{d \mathbf{u}_{\perp}}{d t}=\left[\mathbf{j}_{\perp} \times \mathbf{B}\right]$, where $\rho$ is the mass density.

We define right-handed Cartesian coordinates with the $\mathrm{x}$ and z-axes along $\mathbf{E}_{\perp}$ and $\mathbf{B}$, and put the y-axis to complete the triad.

The solution for the problem (2-4) with zero velocity at the moment $t=0$ is given by the following formula,

$$
\left(\begin{array}{l}
u_{x} \\
u_{y}
\end{array}\right)=\left(\begin{array}{c}
0 \\
-u_{0}
\end{array}\right)+u_{0} e^{-t / \tau}\left(\begin{array}{c}
\sin (t / T) \\
\cos (t / T)
\end{array}\right), u_{z}=0,
$$

where $\mathbf{u}_{0}=\left[\mathbf{E}_{\perp} \times \mathbf{B}\right] / B^{2}$ is the drift velocity, $u_{0}=\left|\mathbf{u}_{0}\right|$, and the parameters $\tau$ and $T$ are

$\tau=\rho /\left(B^{2} \sigma_{P}\right), \quad T=\rho /\left(B^{2} \sigma_{H}\right)$.

In the $\left(u_{x}, u_{y}\right)$ plane this solution is presented by a spiral. It starts at the zero point and goes to the point $\left(0,-u_{0}\right)$ which corresponds to the drift. The direction of this spiral rotation is defined by the sign of $\sigma_{H}$. Let $\sigma_{H}>0$ for definiteness.

As a consequence of the Eqs. $(2,3,5)$, the current density varies with time,

$$
\begin{aligned}
\mathbf{j}_{\perp}= & \left(\sigma_{P} \cos (t / T)+\sigma_{H} \sin (t / T)\right) e^{-t / \tau} \mathbf{E}_{\perp}- \\
& -\left(-\sigma_{P} \sin (t / T)+\sigma_{H} \cos (t / T)\right) e^{-t / \tau}\left[\mathbf{E}_{\perp} \times \mathbf{B}\right] / B .
\end{aligned}
$$

By analogy with the law (Eq. 2), this equality may be interpreted as an Ohm's law in the laboratory frame of reference with the following time varying values of the conductivity tensor components,

$$
\left(\begin{array}{c}
\sigma_{P}(t) \\
\sigma_{H}(t)
\end{array}\right)=e^{-t / \tau}\left(\begin{array}{cc}
\cos (t / T) & \sin (t / T) \\
-\sin (t / T) & \cos (t / T)
\end{array}\right)\left(\begin{array}{c}
\sigma_{P} \\
\sigma_{H}
\end{array}\right) .
$$

If the process under analysis covers time $t_{0}$, it is natural to define an average value of the conductivity. Time integration of the formula (7) and rearrangement with $\tau / T=\sigma_{H} / \sigma_{P}$ gives

$$
\begin{aligned}
& <\sigma_{P}>=\sigma_{P} \frac{\tau}{t_{0}}\left(1-\exp \left(-t_{0} / \tau\right)\right), \\
& <\sigma_{H}>=\sigma_{H}\left[\frac{T}{t_{0}} \sin \frac{t_{0}}{T}\right] \exp \left(-t_{0} / \tau\right) .
\end{aligned}
$$

When $t_{0} \ll \tau, T$, the average values are equal to the original ones. For long-term processes $t_{0} \gg \tau, T$, the average conductivities $\left\langle\sigma_{P}\right\rangle$ and $\left\langle\sigma_{H}\right\rangle$ go to zero. As it is shown in the next sections, the typical situation in the Earth's ionosphere is $T \gg t_{0}$. This permits to simplify Eq. (9),

$$
<\sigma_{H}>\simeq \sigma_{H} \exp \left(-t_{0} / \tau\right)
$$

Then, only the $t_{0} / \tau$ ratio defines $\left\langle\sigma_{P}\right\rangle$ and $\left\langle\sigma_{H}\right\rangle$ modification. The average Hall conductivity $\left\langle\sigma_{H}\right\rangle$ decreases as the exponent of the duration of the process $t_{0}$. The average Pedersen conductivity $\left\langle\sigma_{P}\right\rangle$ varies twice less than $\left\langle\sigma_{H}\right\rangle$ does, when $t_{0}$ is small, and decreases as $1 / t_{0}$, when $t_{0} \gg \tau$. Anyway, the conductivities vanish because of the conductor acceleration if the process is long enough.

The next section presents the empirical models of ionospheric parameters which we use. Then, the effect of the ionospherical conductor motion is analyzed under typical conditions. 


\section{Empirical model of the main ionospheric parameters}

Our calculations are based on the following empirical models: The International Reference 2001 (IRI), the Mass Spectrometer Incoherent Scatter 1990 E (MSISE), the International Geomagnetic Reference Field 1945-2010 (IGRF-10). We used Fortran software of these models from the web page of NASA's Space Physics Data Facility (NASA, 2006).

The concentration of the main ions $\mathrm{O}^{+}, \mathrm{O}_{2}^{+}, \mathrm{NO}^{+}$, and electrons, as well as their temperatures, we define by IRI. To include the height region of $80-100 \mathrm{~km}$ we choose the model (Danilov and Smirnova, 1995) among those included to IRI. All other alternatives inside IRI are solved as a choice of standard versions. The main neutral gases $\mathrm{N}_{2}, \mathrm{O}_{2}, \mathrm{O}$ concentrations, we define by the MSISE model (Hedin, 1991). Above $120 \mathrm{~km}$ this model does not differ from MSIS (Hedin et al., 1977).

The model IRI demonstrates the convergence of electron, ions and neutrals temperatures, when the height decreases to $120 \mathrm{~km}$. This permits one to define a common temperature. We use the MSISE model to define the temperature below $120 \mathrm{~km}$ because it can not be done by IRI.

The collision rates of the electrons can be calculated by the following formulae (Banks, 1966)

$$
\begin{aligned}
v\left(e, \mathrm{O}_{2}\right) & =1.82 \times 10^{-16} n\left(\mathrm{O}_{2}\right)\left(1+0.036 \sqrt{T_{e}}\right) \sqrt{T_{e}}, \\
v\left(e, \mathrm{~N}_{2}\right) & =2.33 \times 10^{-17} n\left(\mathrm{~N}_{2}\right)\left(1-0.000121 T_{e}\right) T_{e}, \\
v(e, 0) & =2.8 \times 10^{-16} n(\mathrm{O}) \sqrt{T_{e}}, \\
v_{e i} & =54 \times 10^{-6} n_{e} T_{e}^{-1.5}, \\
v_{e} & =v_{e i}+v\left(e, \mathrm{O}_{2}\right)+v\left(e, \mathrm{~N}_{2}\right)+v(e, 0),
\end{aligned}
$$

where $n\left(\mathrm{O}_{2}\right)$ is the concentration of the molecules $\mathrm{O}_{2}$ etc., $n_{e}$ and $T_{e}$ are the density and Kelvin temperature of electrons, and $v_{e}$ is the total electron collision rate. For the ions - neutrals collisions, we use the formulae (Stubbe, 1968)

$$
\begin{aligned}
v\left(\mathrm{O}^{+}\right)= & 1.86 \times 10^{-15}\left(\frac{T_{i}+T_{n}}{2000}\right)^{0.37} n(\mathrm{O})+ \\
& +10^{-15} n\left(\mathrm{O}_{2}\right)+1.08 \times 10^{-15} n\left(\mathrm{~N}_{2}\right), \\
v\left(\mathrm{O}_{2}{ }^{+}\right)= & 1.17 \times 10^{-15}\left(\frac{T_{i}+T_{n}}{2000}\right)^{0.28} n\left(\mathrm{O}_{2}\right)+ \\
& +0.75 \times 10^{-15} n(\mathrm{O})+0.89 \times 10^{-15} n\left(\mathrm{~N}_{2}\right), \\
v\left(\mathrm{NO}^{+}\right)= & 0.83 \times 10^{-15} n\left(\mathrm{O}_{2}\right)+ \\
& +0.76 \times 10^{-15} n(0)+0.90 \times 10^{-15} n\left(\mathrm{~N}_{2}\right) .
\end{aligned}
$$

The parameters given by the IRI model and Eqs. $(11,12)$ are sufficient to calculate the components of the conductivity tensor by the following formulae (see, e.g. Hargreaves, 1979),

$$
\begin{aligned}
\sigma_{\|} & =e^{2} N\left(\frac{1}{m_{e} v_{e}}+\sum_{i} \frac{1}{m_{i} v_{i}}\right), \\
\sigma_{P} & =e^{2} N\left(\frac{v_{e}}{m_{e}\left(\omega_{e}^{2}+v_{e}^{2}\right)}+\sum_{i} \frac{v_{i}}{m_{i}\left(\omega_{i}^{2}+v_{i}^{2}\right)}\right),
\end{aligned}
$$

$\sigma_{H}=e^{2} N\left(\frac{\omega_{e}}{m_{e}\left(\omega_{e}^{2}+v_{e}^{2}\right)}-\sum_{i} \frac{\omega_{i}}{m_{i}\left(\omega_{i}^{2}+v_{i}^{2}\right)}\right)$,

where $\omega_{e}$ and $\omega_{i}$ are the electron and ions gyrofrequencies, $m_{e}$ and $m_{i}$ are their masses. The summation is over the main ions $\mathrm{O}^{+}, \mathrm{O}_{2}^{+}, \mathrm{NO}^{+}, i=1,2,3$.

\section{Typical acceleration periods}

The above presented set of models permits us to calculate spatial distributions of ionospheric parameters for any date and time. Equations (6) give the values of the parameters $\tau$ and $T$ which represent the period of a conductor acceleration, which is also the period of the conductivity relaxation to the zero value.

For definiteness we choose a moment of universal time when there is midnight at the Northern geomagnetic pole on the 90-th day of a year that corresponds to the Spring equinox. Other conditions are the following: low geomagnetic activity, the $A_{p}$ index equals 4 , moderate Solar activity, the Covington index equals 130.

The vertical cross-sections of $\tau$ and $T$ above the points with geomagnetic latitudes $\theta_{m}=90^{\circ}, 45^{\circ}$ and longitudes $\varphi_{m}=180^{\circ}, \varphi_{m}=0^{\circ}$ are presented in Fig. 1. Here $h$ is the height from the Earth's surface. We use these points as typical ones for the low latitude and middle latitude ionosphere. There is midnight at the points with $\varphi_{m}=0^{\circ}$ and midday at the points with $\varphi_{m}=180^{\circ}$.

The $\tau$ and $T$ height distributions moderately vary with $\theta_{m}$, $\varphi_{m}$ in the day-side ionosphere. In the height region of 160 $300 \mathrm{~km}$, both parameters are much larger in the middle latitude ionosphere than near the equator as it can be seen by comparison of Fig. 1d and b.

A typical time of a quasi-stationary process in the Earth's ionosphere may be defined as $t_{0}=10^{4} \mathrm{~s}$ because of daily variations.

As it can be seen in Fig. 1, there is $T \geq 10^{4} \mathrm{~s}$ elsewhere. The value of the factor in the bracket in the expression (9) does not differ much from unity if $T>t_{0}$,

$1 \geq \frac{T}{t_{0}} \sin \frac{t_{0}}{T} \geq \sin 1 \simeq 0.85$,

and it goes to unity when $T$ increases.

The parameter $T$ decreases down to $10^{4} \mathrm{~s}$ only in a thin layer near $140 \mathrm{~km}$ in the daytime ionosphere. There is simultaneously $\tau \simeq 10^{4} \mathrm{~s}$ in this region. Therefore, the conductivity $\sigma_{H}$ varies more strongly with $\tau$ through the factor $\exp \left(-t_{0} / \tau\right)$ than it does with $T$ by Eq. (14). It is four times more for $t_{0} \sim 10^{4} \mathrm{~s}$. In the nighttime ionosphere, $T \gg 10^{4} \mathrm{~s}$. Therefore, we neglect the functional behavior on $T$ all over the ionosphere and use the simplified Eq. (10) instead of Eq. (9) for the processes of interest whose typical time $t_{0}$ does not exceed $10^{4} \mathrm{~s}$. 

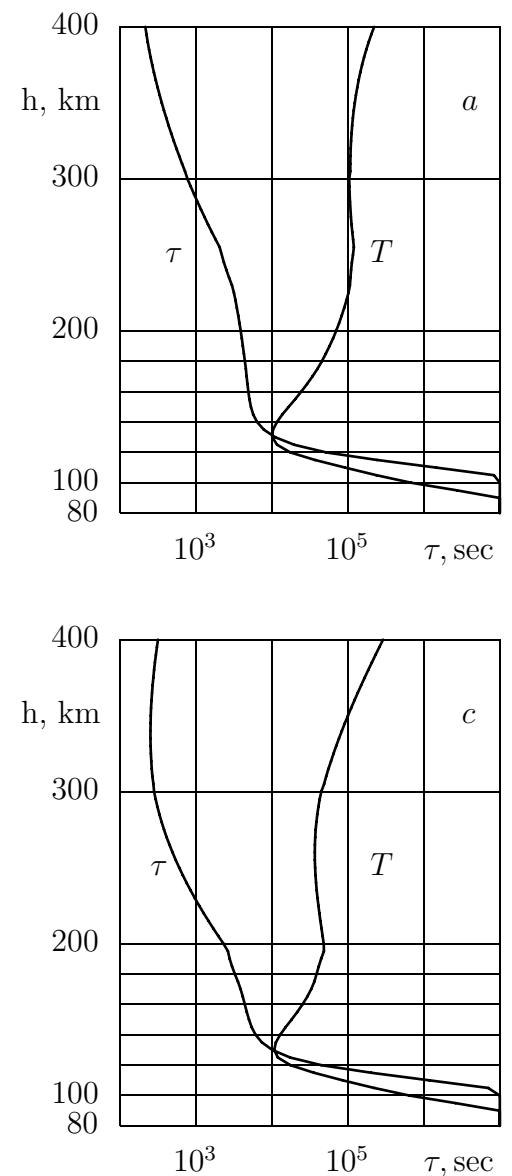
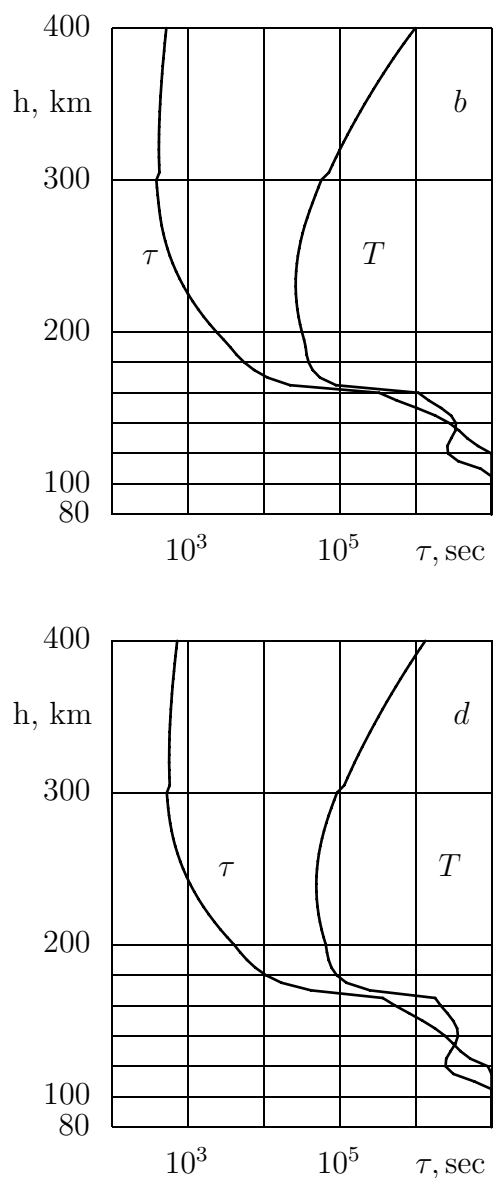

Fig. 1. Vertical cross-sections of the time parameters $\tau$ and $T$. The panels (a), (b) correspond to $\theta_{m}=90^{\circ},(\mathbf{c}),(\mathbf{d})$ correspond to $\theta_{m}=45^{\circ}$. The left and right columns present midday and midnight values, respectively.

The parameter $\tau$ decreases with height. It equals three hours at the height of about $140 \mathrm{~km}$ at midday and at 170 $250 \mathrm{~km}$ at midnight. Since the functions $\tau(z)$ monotonously decrease with height for every fixed $\theta_{m}, \varphi_{m}$ there is a point $z\left(\theta_{m}, \varphi_{m}\right)$ in which $\tau=t_{0}$. The set of these points taken for all values of $\theta_{m}, \varphi_{m}$ forms some surface. This surface divides the ionosphere into two regions. The motion of the ionospheric medium is almost independent of the electric field below the surface. The effective conductivity $(8,9)$ is correspondingly close to the original $\sigma_{P}, \sigma_{H}$. This region is referred as the dynamo region (Hargreaves, 1979). Above this surface, the medium is quickly accelerated up to the drift velocity and its effective conductivity decreases significantly. Equations $(8,9)$ estimate such a separation quantitatively. It depends on the typical period of the process under consideration, $t_{0}$.

The curves 1 in Fig. 2 show the height distributions of $\sigma_{P}, \sigma_{H}$ for the same conditions as Fig. 1, respectively. The panels (a), (c), (e), (g) are plotted for midday and the panels (b), (d), (f), (h) present midnight distributions. Curves 2, 3, 4 show the effective conductivities $\left\langle\sigma_{P}\right\rangle,\left\langle\sigma_{H}\right\rangle$ (Eqs. 8,
9) which are calculated for the processes with $t_{0}=1 / 3,1,3 \mathrm{~h}$, respectively.

Height distributions of $\sigma_{\|}$are partially plotted at the same panels as $\sigma_{P}$ to demonstrate that $\sigma_{\|}$is thousands times larger than $\sigma_{P}$ above $100 \mathrm{~km}$.

The effective Pedersen conductivity is smaller than the original $\sigma_{P}$ by a factor of ten above $300 \mathrm{~km}$. However, its decrease by a factor of two in the region of the maximum value is more important for two-dimensional models of the ionospheric conductor.

The effective Hall conductivity $\left\langle\sigma_{H}\right\rangle$ decreases much more in comparison to $\sigma_{H}$ than $\left\langle\sigma_{P}\right\rangle$ does in comparison to $\sigma_{P}$. It occurs above $200 \mathrm{~km}$ where even the original $\sigma_{H}$ is small.

Figure $2 b, d, f, h$ shows that the effective conductivities during night time are also significantly less than their original values when $t_{0}$ is large enough.

We analyze the modification of the integrated conductivities in the next section. 

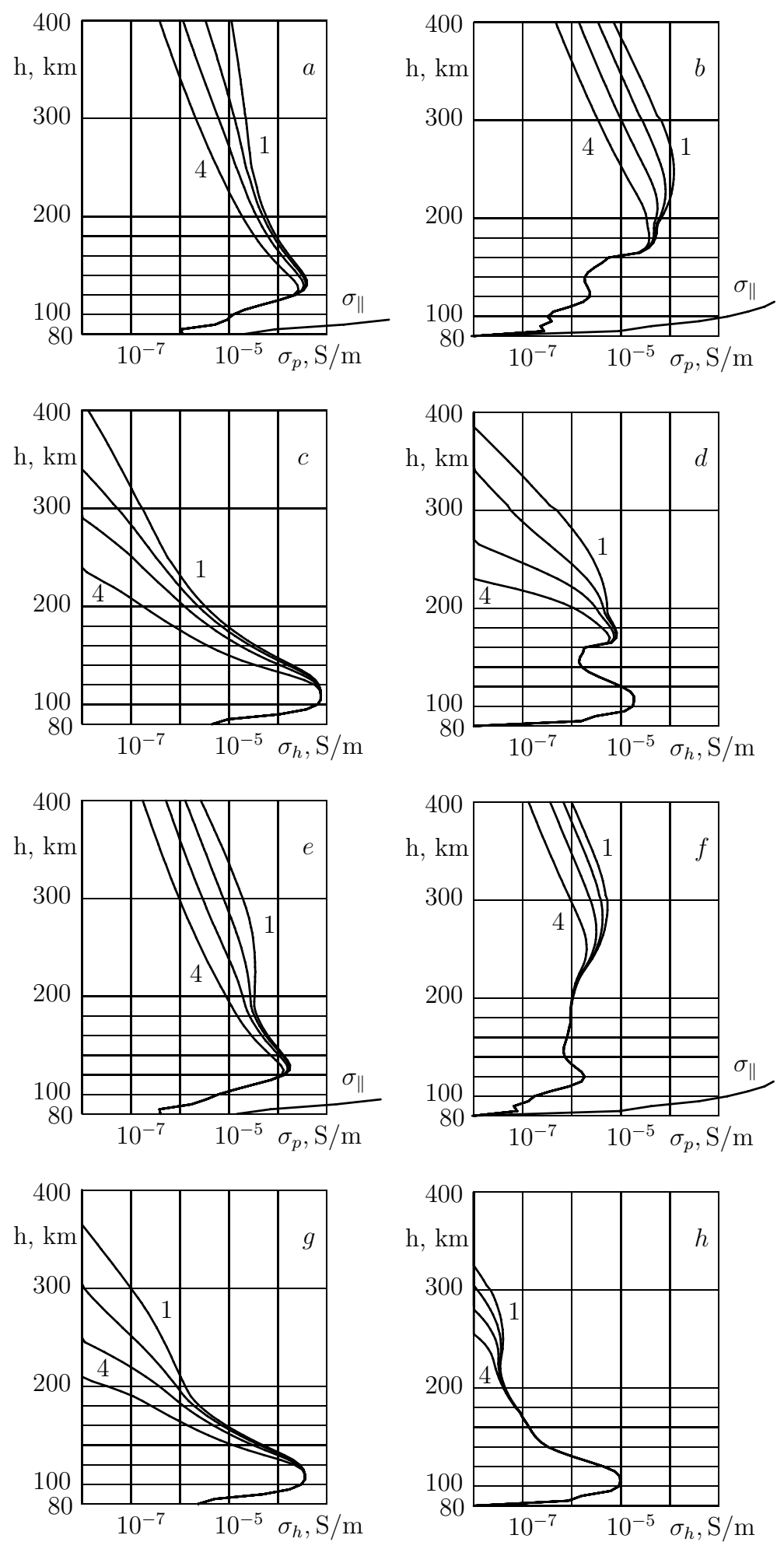

Fig. 2. Height distributions of $\sigma_{P}, \sigma_{H}$ (curves 1), and effective conductivities $\left\langle\sigma_{P}\right\rangle,\left\langle\sigma_{H}\right\rangle$ for $t_{0}=1 / 3,1,3 \mathrm{~h}$ (curves $2,3,4$ ). The conditions are the same as for Fig. 1. The panels (a), (b), (c), (d) correspond to $\theta_{m}=90^{\circ},(\mathbf{e}),(\mathbf{f}),(\mathbf{g}),(\mathbf{h})$ correspond to $\theta_{m}=45^{\circ}$. The left and right columns present midday and midnight values, respectively. Height distributions of $\sigma_{\|}$are partially plotted at the same panels as $\sigma_{P}$. 


\section{2-D model of the ionospherical global conductor}

Considering the high conductivity in the direction of the magnetic field, a two-dimensional approach is appropriate. The magnetic field lines are equipotentials and the ionospheric conductor may be represented by Pedersen and Hall conductances which are equal to integrals of the corresponding local conductivities $\sigma_{P}, \sigma_{H}$ (Hargreaves, 1979). We follow the approach by Gurevich et al. (1976) where this procedure is made accurately from the mathematical point of view by introducing some base surface that crosses all magnetic field lines and it is used both, for numbering of the field lines and as a fictitious 2-D conductor film that is equivalent to a real space distributed conductor.

Here we present a simplified version of the approach by Gurevich et al. (1976). Such a simplification is possible when magnetic field is normal to the base surface. For a potential magnetic field $\mathbf{B}=-\operatorname{grad} \Phi$. Any surface $\Phi=$ const may be used as such a base surface since the vector grad $\Phi$ is always normal to the surface $\Phi=$ const. We use the empirical model IGRF that represents just the potential part of the geomagnetic field. It would be impossible if it includes magnetic perturbations created by the currents in the magnetosphere. An additional simplification is possible since we use semi geodetic coordinates on the base surface. Since the surface $\Phi=$ const is smooth such a coordinate system exists (Korn and Korn, 1968).

The conductivity is a scalar in the atmosphere below $80 \mathrm{~km}$ and it is a tensor with the $\sigma_{P}, \sigma_{H}, \sigma_{\|}$components in the ionosphere which can be calculated by Eq. (13).

Let us suppose that the conductor does not move. Then the Ohm law (Eq. 1) stays for the parallel components and the law (2) is simplified for the normal ones,

$\mathbf{j}_{\perp}=\left(\begin{array}{cc}\sigma_{P} & -\sigma_{H} \\ \sigma_{H} & \sigma_{P}\end{array}\right) \mathbf{E}_{\perp}$.

In Fig. 2 it is demonstrated how the difference between $\sigma_{\|}$ and $\sigma_{P}$ increases in the height region $80-100 \mathrm{~km}$. Since $\sigma_{\|}$ in the ionosphere is thousands times larger than $\sigma_{P}, \sigma_{H}$ it is possible to idealize this inequality as

$\sigma_{\|}=\infty$

The conductivities $\sigma_{P}, \sigma_{H}$ are small below $80 \mathrm{~km}$ and can not make a substantial contribution to the integrals. Moreover, the two-dimensional approximation fails there because the field-aligned conductivity $\sigma_{\|}$becomes comparable to $\sigma_{P}$. The approximation $\sigma_{\|}=\infty$ is satisfactory above $80 \mathrm{~km}$, if electric fields above $100 \mathrm{~km}$ are under analysis (Stening, 1985). If electric fields and currents below $100 \mathrm{~km}$ are of interest, it would be necessary to solve another conductivity problem in some domain below the new boundary about $100 \mathrm{~km}$ taking obtained potential distribution at this boundary.
The equality (16) means that the electric current along a magnetic field line can be arbitrary while the electric field component $E_{\|}$equals zero,

$E_{\|}=0$.

Since $\mathbf{E}=-\operatorname{grad} V$, the electric potential $V$ is constant at each magnetic field line as a result of the identity (17) and

$\mathbf{E}_{\perp}=-\operatorname{grad}_{\perp} V$.

In such a model, a magnetic field line is an object with its own value of the electric potential $V$. It can obtain or loose charge by currents $\mathbf{j}_{\perp}$ and it does not matter for its total charge in what point of the magnetic field line $\mathbf{j}_{\perp}$ exists because charge can go free along this line according to Eq. (16).

Denote the semi geodetic coordinates at the base surface as $m, h$. Then the distance between two points at the base surface which are close to each other equals

$g^{2}(\Delta m)^{2}+(\Delta h)^{2}$,

where the components of the metric tensor are $g^{2}, 1$. The procedure of such a coordinate system construction is presented in (Korn and Korn, 1968). The third coordinate $l$ is the arc length along the magnetic field line from the point at the base surface.

In purpose to define the conductance for a magnetic field line let us analyze a thin magnetic field tube that has a square cross-section in some plane normal to the magnetic field. The cross-section is shown in Fig. 3 with the $l$ axis directed out of the paper. We put magnetic field lines from the point 0 till some point $0^{\prime}$. We construct local Cartesian coordinates near the point $0^{\prime}$ with the $\mathrm{z}$-axis along magnetic field. We put the $x$-axis from the point $0^{\prime}$ to point $0^{\prime}$ in that the field line from the point 1 intersects the plane $z=0$. We put the $y$-axis to complete the triad. We also design the magnetic field line from the point 2 till it crosses the plane $z=0$ in some point $2^{\prime}$.

Since we analyze a thin magnetic field tube with small $g \Delta m=\Delta h$ we get some parallelogram in the plane $z=0$ as the projection along magnetic field lines of the quadrangle into the plane $l=0$. This is shown by the right-hand panel in Fig. 3. We denote the coordinates of the points $1^{\prime}, 2^{\prime}$ as

$\left(\Delta x_{1}, 0,0\right), \quad\left(\Delta x_{2}, \Delta y_{2}, 0\right)$,

with zero point $0^{\prime}$ coordinates by construction.

Because of the expression (18), the potential differences between the points 1, 0 and between 2, 0 are equal to

$\Delta V_{1}=-E_{m} g \Delta m, \quad \Delta V_{2}=-E_{h} \Delta h$

and between the points $1^{\prime}, 0^{\prime}$ and between $2^{\prime}, 0^{\prime}$

$\Delta V_{1}^{\prime}=-E_{x} \Delta x_{1}, \quad \Delta V_{2}^{\prime}=-E_{x} \Delta x_{2}-E_{y} \Delta y_{2}$.

Here we take advantage of the fact that the cross-sections of the magnetic field tube are so small that the electric field may be taken as homogeneous across the tube and the Eq. (20). 

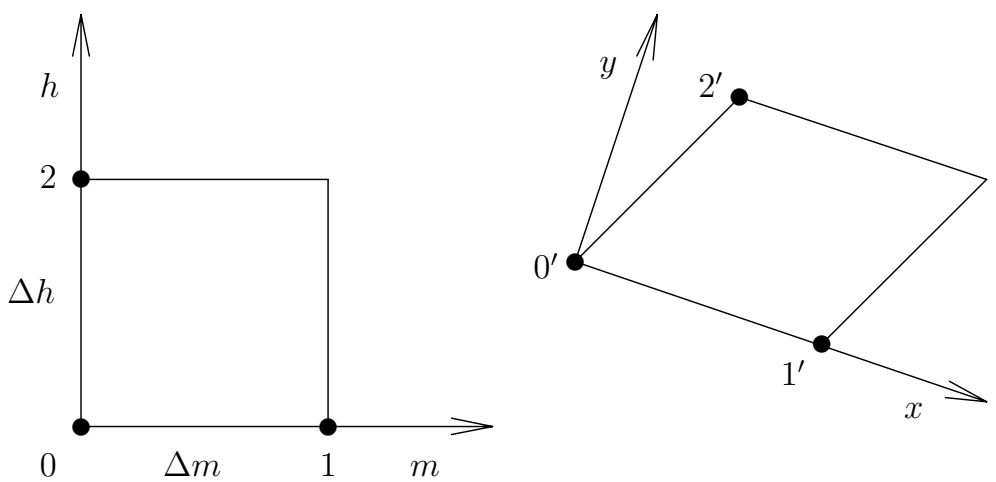

Fig. 3. Normal cross-sections of a magnetic field tube which starts at the $m, h$ plane. The right panel presents another cross-section of the same tube. The local Cartesian coordinates $x, y, z$ are introduced near the point $0^{\prime}$.

The potential differences $\Delta V_{1}, \Delta V_{2}$ (Eq. 21) are the same as $\Delta V_{1}^{\prime}, \Delta V_{2}^{\prime}$ (Eq. 22) because the values of the potential at the points 0 and $0^{\prime}, 1$ and $1^{\prime}, 2$ and $2^{\prime}$ are equal since these pairs belong to the same field lines. This permits to calculate the electric field in the plane $z=0$ from the last formulae,

$$
\left(\begin{array}{c}
E_{x} \\
E_{y}
\end{array}\right)=\left(\begin{array}{cc}
\frac{g \Delta m}{\Delta x_{1}} & 0 \\
-\frac{g \Delta m \Delta x_{2}}{\Delta y_{2} \Delta x_{1}} & \frac{\Delta h}{\Delta y_{2}}
\end{array}\right)\left(\begin{array}{c}
E_{m} \\
E_{h}
\end{array}\right) .
$$

This electric field $\mathbf{E}_{\perp}$ produces the current $\mathbf{j}_{\perp}$ by Ohm's law (Eq. 15),

$$
\left(\begin{array}{l}
j_{x} \\
j_{y}
\end{array}\right)=\left(\begin{array}{cc}
\sigma_{P} & -\sigma_{H} \\
\sigma_{H} & \sigma_{P}
\end{array}\right)\left(\begin{array}{c}
E_{x} \\
E_{y}
\end{array}\right) .
$$

Let us consider a prism $0<z<\Delta z$ and currents $\Delta I_{1}, \Delta I_{2}$ through two of its sides which have height $\Delta z$ and contain the edges $0^{\prime} 1^{\prime}$ and $0^{\prime} 2^{\prime}$, correspondingly. We must multiply the normal components of current density (Eq. 24) with the squares of these rectangle sides,

$$
\left(\begin{array}{l}
\Delta I_{2} \\
\Delta I_{1}
\end{array}\right)=\Delta z\left(\begin{array}{cc}
\Delta y_{2} & -\Delta x_{2} \\
0 & \Delta x_{1}
\end{array}\right)\left(\begin{array}{l}
j_{x} \\
j_{y}
\end{array}\right) .
$$

The same currents through the sides of the analyzed magnetic tube may be provided by some surface current density $\Delta \mathbf{J}$ at a fictitious thin conducting film in the plane $l=0$ if the components $\Delta J_{m}, \Delta J_{h}$ of $\Delta \mathbf{J}$ are such that

$\Delta I_{2}=\Delta J_{m} \Delta h, \quad \Delta I_{1}=\Delta J_{h} g \Delta m$.

Taking into account Eqs. (23-26), we can express

$$
\begin{aligned}
\left(\begin{array}{c}
\Delta J_{m} \\
\Delta J_{h}
\end{array}\right)=\Delta & z\left(\begin{array}{cc}
\frac{\Delta y_{2}}{\Delta h} & -\frac{\Delta x_{2}}{\Delta h} \\
0 & \frac{\Delta x_{1}}{g \Delta m}
\end{array}\right)\left(\begin{array}{cc}
\sigma_{P} & -\sigma_{H} \\
\sigma_{H} & \sigma_{P}
\end{array}\right) \times \\
& \times\left(\begin{array}{cc}
\frac{g \Delta m}{\Delta x_{1}} & 0 \\
-\frac{g \Delta m \Delta x_{2}}{\Delta y_{2} \Delta x_{1}} & \frac{\Delta h}{\Delta y_{2}}
\end{array}\right)\left(\begin{array}{c}
E_{m} \\
E_{h}
\end{array}\right) .
\end{aligned}
$$

This means that the conducting prism $0<z<\Delta z$ can be replaced by a fictitious conducting film in the plane $l=0$ with conductance tensor $\Delta \Sigma$ that is the coefficient in front of the vector $\left(E_{m}, E_{h}\right)$ in Eq. (27)

$$
\Delta \Sigma=\Delta z\left(\begin{array}{cc}
\frac{\Delta x_{2}^{2}+\Delta y_{2}^{2}}{\Delta x_{1} \Delta y_{2}} \sigma_{P} & -\frac{\Delta x_{2}}{\Delta y_{2}} \sigma_{P}-\sigma_{H} \\
-\frac{\Delta x_{2}}{\Delta y_{2}} \sigma_{P}+\sigma_{H} & \frac{\Delta x_{1}}{\Delta y_{2}} \sigma_{P}
\end{array}\right),
$$

where the equality $g \Delta m=\Delta h$ is used.

By summation of the incomes from all cross-sections of the magnetic field tube, we obtain the conductance of such a film in the plane $l=0$ which, in respect of currents exchange between magnetic field lines, is equivalent to the surrounding of the whole magnetic field line. The resulting Ohm law can be written as $\mathbf{J}=\Sigma \mathbf{E}$, or in detailed form as

$$
\left(\begin{array}{c}
J_{m} \\
J_{h}
\end{array}\right)=\left(\begin{array}{cc}
\Sigma_{m m} & \Sigma_{m h} \\
\Sigma_{h m} & \Sigma_{h h}
\end{array}\right)\left(\begin{array}{c}
E_{m} \\
E_{h}
\end{array}\right) \text {. }
$$

Since the coordinate $l$ is the length along this magnetic field line it can be used as a local Cartesian coordinate $d l=d z$ near the plane $z=0$. After integration of Eq. (28), we get

$$
\Sigma=\int\left(\begin{array}{cc}
\frac{\Delta x_{2}^{2}+\Delta y_{2}^{2}}{\Delta x_{1} \Delta y_{2}} \sigma_{P} & -\frac{\Delta x_{2}}{\Delta y_{2}} \sigma_{P}-\sigma_{H} \\
-\frac{\Delta x_{2}}{\Delta y_{2}} \sigma_{P}+\sigma_{H} & \frac{\Delta x_{1}}{\Delta y_{2}} \sigma_{P}
\end{array}\right) d l .
$$

We see that only ratios of $\Delta x_{1}, \Delta x_{2}, \Delta y_{2}$ are present and they are known functions of $l$ because the magnetic field lines are proposed to be known as well as the $\sigma_{P}, \sigma_{H}$ space distributions.

If the magnetic field lines are parallel straight lines, then

$$
\Delta y_{2} / \Delta x_{1}=1, \quad \Delta x_{2}=0 .
$$

Therefore Eq. (30) is simplified to

$$
\Sigma=\int\left(\begin{array}{cc}
\sigma_{P} & -\sigma_{H} \\
\sigma_{H} & \sigma_{P}
\end{array}\right) d l
$$

what permits to write down the tensor $\Sigma$ as

$$
\Sigma=\left(\begin{array}{cc}
\Sigma_{P} & -\Sigma_{H} \\
\Sigma_{H} & \Sigma_{P}
\end{array}\right)
$$




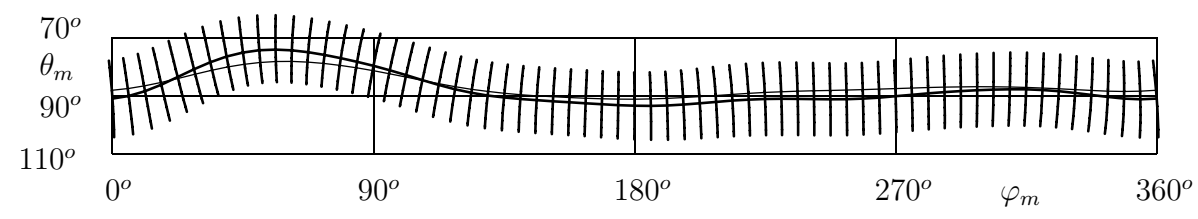

Fig. 4. Geomagnetic equator with segments of the magnetic field lines which start and finish at the height of $80 \mathrm{~km}$, and cross the geomagnetic equator at the height of $400 \mathrm{~km}$. A possible base surface near the geomagnetic equator is plotted by the thin line.

with Pedersen and Hall conductances $\Sigma_{P}, \Sigma_{H}$ which are obtained from the local Pedersen and Hall conductivities $\sigma_{P}, \sigma_{H}$ integrated along a magnetic field line. If a segment of the magnetic field line with nonzero $\sigma_{P}, \sigma_{H}$ is short enough, then the equalities (31) are approximately valid for any magnetic field. This is so in the high and middle latitude ionosphere where the magnetic field lines cross the ionospheric conducting layer with some angle $\xi$ from the vertical that increase the length as $\Delta l=\Delta H / \cos \xi$, where $\Delta H$ is the vertical size of the layer.

It is necessary to emphasize that such a tensor $\Sigma$ with only $\Sigma_{P}, \Sigma_{H}$ components stays only in coordinates $m, h$ normal to the magnetic field.

Since the parameters of the ionosphere do not vary much in the horizontal direction, the integrals (32) can be calculated as height integrals in high latitudes and just height integrals are often referred as to integral conductivities (Hargreaves, 1979), but there is no reason to do so in low latitudes since the electric field is constant not with height but along magnetic field lines which are almost horizontal.

We use the IGRF model for the geomagnetic field. Figure 4 presents the position of the geomagnetic equator at the height $400 \mathrm{~km}$ and a possible base surface at the same height. They are presented by thick and thin lines, respectively. The abscissa is the geomagnetic longitude $\varphi_{m}$ and the ordinate is the geomagnetic colatitude $\theta_{m}$. Both lines vary with height because of the non-dipolar part of the geomagnetic field, but, the variations are hardly seen in the figure for heights of 80 $400 \mathrm{~km}$. The segments of the magnetic field lines are also plotted. These lines cross the geomagnetic equator at the height of $400 \mathrm{~km}$. The ends of the segments lie at a height of $80 \mathrm{~km}$.

The geomagnetic equator is the line of the horizontal magnetic field and the base surface has constant value of the magnetic potential. We mention that the magnetic field is not normal to the geomagnetic equator in nondipolar models and the base surface is defined so that the magnetic field is normal to it. Any equipotential surface may be used as the base surface. Here we have chosen the surface that gives the best values in the following estimations.

We have calculated the magnetic field lines in the neighborhood of the lines presented in Fig. 4 that permitted us to obtain the values of the geometrical coefficients in the integral (30) and to find that they satisfy the inequalities

$$
\begin{aligned}
& \left|\frac{\Delta x_{2}^{2}+\Delta y_{2}^{2}}{\Delta x_{1} \Delta y_{2}}-1\right|<0.30, \\
& \left|\frac{\Delta x_{2}}{\Delta y_{2}}\right|<0.21, \quad\left|\frac{\Delta x_{1}}{\Delta y_{2}}-1\right|<0.43 .
\end{aligned}
$$

The lines which start below these lines and finish at the same height of $80 \mathrm{~km}$ satisfy these inequalities as well. The segments of the field lines which are in the height interval 80 $400 \mathrm{~km}$ in the rest ionosphere also satisfy these inequalities if the base surface is normal to the lines and because of that the line segments are shorter than the lengths of those shown in Fig. 4 and the non-dipolar components of the geomagnetic field are not too large. But similar geometrical coefficients additionally appear if we take into account that the Northern and Southern semi-lines must be united by the same potential value at least in low and middle latitudes. It is shown by Denisenko and Zamay (1992) how to construct such a base surface for the whole ionosphere as a set of small sheets and to transform it into a unit circle for a dipolar magnetic field. For the real geomagnetic field this can be done only numerically.

The numbers in the inequalities (34) estimate the error if the expression (30) is simplified with Eq. (32). If we use only the dipolar component of the geomagnetic field, then the base surface and the equator become the plane $\theta_{m}=\pi / 2$ and the calculations give

$$
\begin{aligned}
& \frac{\Delta x_{2}^{2}+\Delta y_{2}^{2}}{\Delta x_{1} \Delta y_{2}}-1 \simeq-0.06, \\
& \frac{\Delta x_{2}}{\Delta y_{2}} \simeq 0.00, \quad \frac{\Delta x_{1}}{\Delta y_{2}}-1 \simeq 0.07 .
\end{aligned}
$$

The shape of a dipolar magnetic field line is known to be

$r=r_{0} \sin ^{2} \theta, \varphi=\varphi_{0}$,

where $r_{0}, \varphi_{0}$ are the coordinates of the point in the equatorial plane and $r, \theta, \varphi$ are the coordinates of any point at this field line. So, the coefficients can be calculated analytically,

$$
\begin{aligned}
& \frac{\Delta x_{2}^{2}+\Delta y_{2}^{2}}{\Delta x_{1} \Delta y_{2}}=\frac{1}{\sqrt{1+3 \cos ^{2} \theta}}, \\
& \frac{\Delta x_{2}}{\Delta y_{2}}=0, \quad \frac{\Delta x_{1}}{\Delta y_{2}}=\sqrt{1+3 \cos ^{2} \theta} .
\end{aligned}
$$


For the lines in the height interval 80-400 km, Eq. (36) gives $|\pi / 2-\theta|<13^{\circ}$ and the values given by Eq. (37) correspond to the numerically obtained ones in the equalities (35).

As it is shown by the estimations $(34,35)$, the simplified definition of the conductances (32) is rather precise for a dipolar field, and it gives an error up to $40 \%$ for the real field. Nevertheless, for simplicity, we use just Pedersen and Hall conductances $\Sigma_{P}, \Sigma_{H}$ near the geomagnetic equator in our further demonstrations of the results of the plasma acceleration, because the effect under analysis decreases the conductivity a few times.

The current density (Eq. 24) satisfies the local charge conservation law

$\operatorname{div} \mathbf{j}=\frac{\partial j_{x}}{\partial x}+\frac{\partial j_{y}}{\partial y}+\frac{\partial j_{z}}{\partial z}=q$,

where nonzero $q$ appears if some given extrinsic current $\mathbf{j}_{e x}$ exists. Then

$q=-\operatorname{div} \mathbf{j}_{e x}$.

For example such an extrinsic current appears in accordance with Ohm's law (Eq. 2)

$\mathbf{j}_{e x}=\left(\begin{array}{cc}\sigma_{P} & -\sigma_{H} \\ \sigma_{H} & \sigma_{P}\end{array}\right)[\mathbf{u} \times \mathbf{B}]$,

when the velocity $\mathbf{u}$ in the ionosphere is given. Such a current source is under analysis in the dynamo theory (Akasofu and Chapman, 1972). Different given currents in the magnetospheric parts of the magnetic field lines also may be taken into account as $\mathbf{j}_{e x}$.

The integral of the local Eq. (38) over the magnetic field tube presented in Fig. 3 from $l_{\text {st }}$ till $l_{\text {fin }}$ is equal to the sum of the currents through its four sides whose cross-sections are presented in Fig. 3 plus currents through the ends. In accordance with the definition of the fictitious current at the base surface, the sum of these four currents is equal to

$\oint\left[\left(J_{m}, J_{h}\right) \times(d m, d h)\right]$.

The current through the end of the magnetic field tube is

$j_{\|} \Delta x_{1} \Delta y_{2}$,

where the field-aligned current density $j_{\|}$and the square of the tube $\Delta x_{1} \Delta y_{2}$ are taken at $l_{\mathrm{st}}$ or at $l_{\mathrm{fin}}$. If we regard the whole tube that starts below the ionosphere and finishes also below the ionosphere in the opposite hemisphere, then $j_{\|}=0$ at the both ends. Otherwise $j_{\|}$at one end of the tube may be nonzero as some given current from the magnetosphere to the ionosphere.

The integral of the right-hand side of Eq. (38) is

$\int_{l_{s t}}^{l_{f i n}} q \Delta x_{1} \Delta y_{2} d l$.

Let us divide the terms (41-43) by the square of the tube at the base surface $g \Delta m \Delta h$ and make the limit $\Delta m \rightarrow 0$,
$\Delta h \rightarrow 0$. We obtain a 2-D operator of the divergence of the integral (41) that in accordance with (Korn and Korn, 1968) is

$\operatorname{Div} \mathbf{J}=\frac{1}{g}\left(\frac{\partial}{\partial m}\left(J_{m}\right)+\frac{\partial}{\partial h}\left(g J_{h}\right)\right)$,

and the function

$Q=\int_{l_{\mathrm{st}}}^{l_{\mathrm{fin}}} q \frac{d x_{1} d y_{2}}{g \Delta m \Delta h} d l-\left.j_{\|} \frac{d x_{1} d y_{2}}{g \Delta m \Delta h}\right|_{l_{\mathrm{st}}} ^{l_{\text {fin }}}$

as the difference between the terms (43) and (42). If the analyzed part of the magnetic field tube is short enough, then the fractions in this expression is equal to unit.

The total result of this integration of the local Eq. (38),

$\operatorname{Div} \mathbf{J}=Q$,

is the charge conservation law for the fictitious current at the base surface.

The Eqs. $(46,18,29)$ give the model of the introduced fictitious conducting film,

$-\operatorname{Div}(\Sigma \operatorname{Grad} V)=Q$,

where the operator Grad produces the $m, h$ components of the full vector grad $V$.

The sharp decrease of the conductance below the ionosphere may be idealized as a jump to zero at some height $h_{1}$. Then, $h=h_{1}$ is the boundary with insulator that means zero normal component of the current density,

$$
\left.J_{h}\right|_{h=h_{1}}=0 .
$$

If we approximately put $\sigma_{P}=\sigma_{H}=0$ at $h<h_{1}$ while integration in Eq. (30), then $\Sigma \rightarrow 0$, when $h \rightarrow h_{1}$. This happens because the length of the segment of the magnetic field line that is inside the layer with $\sigma \neq 0$ goes to zero when the top of the field line goes to the boundary of this layer. Such a singularity $\Sigma \rightarrow 0$ is a bad feature for Eq. (47). To avoid this singularity, we need conductivities $\sigma_{P}, \sigma_{H}$ in some region below $h_{1}$ that makes it possible to calculate nonzero conductance $\Sigma$ at $h \geq h_{1}$.

It is possible to take $h_{1}$ about $80 \mathrm{~km}$ in the daytime ionosphere and $h_{1}$ about $90 \mathrm{~km}$ in the nighttime ionosphere. Less values of $h_{1}$ meet two difficulties. As it can be seen in Fig. 2a, b, quantity $\sigma_{\|}$is not much larger than $\sigma_{P}, \sigma_{H}$ below these heights and so the 2-D model can not be used. The second problem is due to the restrictions of the IRI model. It gives the electron density above $65 \mathrm{~km}$ in the daytime ionosphere and only above $80 \mathrm{~km}$ in the nighttime ionosphere. In the next section, it is demonstrated that these heights include the main part of the ionospheric conductor.

In purpose to plot the conductance $\Sigma$ distributions at the common heights $h>80 \mathrm{~km}$, we extrapolate the electron density below $80 \mathrm{~km}$ in the night time ionosphere as

$n_{e}^{0} \exp \left((h-80 \mathrm{~km}) / h_{0}\right)$, 
where the parameters $n_{e}^{0}, h_{0}$ are defined by the $n_{e}$ values at $h=80 \mathrm{~km}$ and $h=85 \mathrm{~km}$ at every vertical line. The Eq. (49) permits to represent a sharp decrease of the values $\Sigma_{P}, \Sigma_{H}$ below $h=80 \mathrm{~km}$ without their jumps to zero at $h=80 \mathrm{~km}$, that would be if we were to put $n_{e}=0$ and hence $\sigma_{P}=\sigma_{H}=0$ below $h=80 \mathrm{~km}$.

The Eq. (47) must be completed by the boundary condition (48) and some conditions for other boundaries. In the dipolar magnetic field with zero electric potential difference between adjoint points in the Northern and Southern Hemispheres the base surface can be chosen as the plane $\theta_{m}=90^{\circ}$ and the cylindrical coordinates $r, \varphi$ can be used as $h, m$ with metrical $g=r$ in Eq. (19). Then condition (48) becomes

$$
\left.\left(-\Sigma_{r r} \frac{\partial V}{\partial r}-\Sigma_{r \varphi} \frac{1}{r} \frac{\partial V}{\partial \varphi}\right)\right|_{r=R_{E}+h_{1}}=0,
$$

and the condition, that means absence of a singularity near the magnetic poles,

$$
\left.V\right|_{r \rightarrow \infty}=0 \text {, }
$$

completes the model.

Equation (47) takes the shape

$$
\begin{aligned}
-\frac{1}{r} & \frac{\partial}{\partial r}\left(r \Sigma_{r r} \frac{\partial V}{\partial r}+\Sigma_{r \varphi} \frac{\partial V}{\partial \varphi}\right)- \\
& -\frac{1}{r} \frac{\partial}{\partial \varphi}\left(\Sigma_{\varphi r} \frac{\partial V}{\partial r}+\Sigma_{\varphi \varphi} \frac{1}{r} \frac{\partial V}{\partial \varphi}\right)=Q,
\end{aligned}
$$

with the tensor $\Sigma$ calculated by Eq. (30).

If the parts of the magnetic field lines are regarded separately for the Northern and Southern Hemispheres, then $Q$ includes given field-aligned currents above the ionosphere in accordance with Eq. (45). The boundary value problem becomes more complicated in such a model (Denisenko, 2002) in comparison to Eqs. (50-52).

\section{Cowling conductance}

The Cowling conductance

$\Sigma_{C}=\Sigma_{P}+\Sigma_{H}^{2} / \Sigma_{P}$

is widely used (Forbes, 1981) in theories of equatorial electrojets. It appears in the models of the boundary layer for the problem defined by Eqs. $(46,18,29,48)$.

As usual, an approximate model of the boundary layer is based on its small width in comparison to typical distance along the boundary. The ratio of these scales is regarded as to be a small parameter, $\varepsilon$. In our case, $\varepsilon=\delta h / \delta m$ since the boundary (48) is the line $h=h_{1}$. Let us now denote $h_{2}$ as the $h$ coordinate of the line, that separates the boundary layer from the main part of the conductor. These lines may be curved in general case, but, here we simplify the procedure (Gurevich et al., 1976) to make it more visual. We neglect the difference of the metric coefficients from Eq. (31). We also neglect the curvature of the Earth's surface and suppose that the base surface is a plane. Then, semi geodetic coordinates $m, h$ may be chosen as Cartesian ones with a vertical direction $h$ and a horizontal direction $m$ along the geomagnetic equator (Korn and Korn, 1968). The metric tensor becomes a unit one in such a case, what means that $g=1$ in Eq. (19). As usual, we don't take into account the charge source inside the boundary layer, which means $Q=0$ in the charge conservation law (Eq. 46).

After these simplifications, Eq. (46) takes shape

$\frac{\partial J_{m}}{\partial m}+\frac{\partial J_{h}}{\partial h}=0$.

In order to compare the scales of $J_{m}, J_{h}$, let us integrate this equation over the quadrangle $h_{1}<h<h_{1}+\delta h$, $m_{1}<m<m_{1}+\delta m$, where the side $h=h_{1}$ belongs to the boundary with insulator (48) and $m=m_{1}$ is an arbitrary point at the boundary. After integration by parts,

$$
\begin{gathered}
\int_{h_{1}}^{h_{1}+\delta h} J_{m}\left(m_{1}+\delta m, h\right) d h-\int_{h_{1}}^{h_{1}+\delta h} J_{m}\left(m_{1}, h\right) d h+ \\
+\int_{m_{1}}^{m_{1}+\delta m} J_{h}\left(m, h_{1}+\delta h\right) d m=0,
\end{gathered}
$$

where the integral over the side $h=h_{1}$ is omitted because it equals zero in view of Eq. (48). Since each integral of a continuous function over a segment equals the length of the segment multiplied by the value of this function in some point inside the segment, this equation may be rewritten and resolved as

$$
\begin{aligned}
& J_{h}\left(m^{*}, h_{1}+\delta h\right)= \\
& \quad=\frac{\delta h}{\delta m}\left(J_{m}\left(m_{1}+\delta m, h^{* *}\right)-J_{m}\left(m_{1}, h^{* * *}\right)\right),
\end{aligned}
$$

where $*$ marks some point inside proper segment.

Since we are interested in a large scale processes when the distance $\delta m$ of a remarkable $J_{m}$ change is much larger than $\delta h$, the ratio in the right-hand side is a small parameter $\varepsilon$. The first order boundary layer approximation corresponds to the idealization of this small parameter, as $\varepsilon=0$, meaning

$J_{h}\left(m^{*}, h_{1}+\delta h\right)=0$.

Since the values of coordinates $m^{*}, h_{1}+\delta h$ describe any point, the vertical component of the current density equals zero inside the boundary layer,

$J_{h}=0$.

Similar analysis of the equation

$\frac{\partial E_{h}}{\partial m}-\frac{\partial E_{m}}{\partial h}=0$

which is equivalent to Eq. (18) in our Cartesian coordinates, shows that the horizontal component of the electric field is independent of the hight $h$ inside the boundary layer,

$E_{m}(m, h)=E_{m}(m)$. 
Since the conductance tensor in the Ohm's law (29) has the shape (33) in our simplified case, we can write Eq. (29) as

$$
\left(\begin{array}{c}
J_{m} \\
J_{h}
\end{array}\right)=\left(\begin{array}{cc}
\Sigma_{P} & -\Sigma_{H} \\
\Sigma_{H} & \Sigma_{P}
\end{array}\right)\left(\begin{array}{c}
E_{m} \\
E_{h}
\end{array}\right) .
$$

In combination with Eqs. $(58,60)$ this means

$$
\begin{array}{r}
J_{m}(m, h)=\Sigma_{P} E_{m}(m)-\Sigma_{H} E_{h}(m, h) \\
0=\Sigma_{H} E_{m}(m)+\Sigma_{P} E_{h}(m, h) .
\end{array}
$$

From the second equation we obtain

$$
E_{h}(m, h)=-\left(\Sigma_{H} / \Sigma_{P}\right) E_{m}(m),
$$

and, resolving the first one, we obtain the main formula of the boundary layer

$$
J_{m}(m, h)=\left(\Sigma_{P}+\Sigma_{H}^{2} / \Sigma_{P}\right) E_{m}(m) .
$$

The coefficient is referred to as the Cowling conductance (53). It relates the components of the current density and electric field which both are normal to the magnetic field and parallel to the boundary. They are along the geomagnetic equator.

Since $E_{m}$ is independent of $h$, it is simple to integrate expression (64) over the height $h$ to get the total current in the boundary layer,

$$
\begin{aligned}
I(m) & =\int_{h_{1}}^{h_{2}} J_{m}(m, h) d h=\left(\int_{h_{1}}^{h_{2}} \Sigma_{C} d h\right) E_{m}(m)= \\
& =A(m) E_{m}(m),
\end{aligned}
$$

where the total conductance of the boundary layer is a function of the coordinate $m$ along the boundary and is denoted as $A(m)$.

Such a boundary layer approach is correct but of no sense if $\Sigma_{C}$ in the vicinity of the boundary is of the same order of magnitude as inside the main part of the conductor. Some small current $I(m)$ is separated in this strip, but it does not help to analyze the global problem. This is not the case in the ionosphere of the Earth and this boundary layer is observed as the equatorial electrojet. Typical distributions of $\Sigma_{C}$ above the geomagnetic equator are presented in Fig. 5e, f. Large values of $\Sigma_{C}$ are seen in the $\delta h \approx 50 \mathrm{~km}$ layer in the midday. This layer is much wider at midnight. Its upper boundary is not shown because the $\Sigma_{C}$ decrease to about $10 \mathrm{~S}$ middle latitude value is rather slow. If we are interested in the total currents in the equatorial electrojets, the integration (65) can be done till $h=150 \mathrm{~km}$ at midday. The night time structure is analyzed in Sect. 8.

If the problem given by Eqs. $(46,18,29,48)$ is solved numerically, the sharp variations of the coefficients in $h$ direction produce difficulties. They can be avoided by separating the boundary layer taking $h=300 \mathrm{~km}$ to make $\Sigma_{P}, \Sigma_{H}$ smooth functions in the rest domain. The Cowling conductance $\Sigma_{C}$ itself is not used there. The boundary layer approximation is correct in this case, since $h_{2}-h_{1} \approx 200 \mathrm{~km}$ is much less then a few thousands kilometers distance along the equator, indeed. Its adequateness looks problematic if $h_{2}-h_{1}$ exceeds $1000 \mathrm{~km}$, but it is not necessary to use a boundary layer approximation for such a wide region.

There are experimental estimations of the width of this boundary layer, which means the vertical size above the geomagnetic equator. The longitudinal component of the electric field is constant for about $400 \mathrm{~km}$ in the vicinity of the geomagnetic equator (Forbes, 1981) that corresponds to Eq. (60). This means that at least one of the two nontrivial base features for the 2-D boundary layer theory (58), (60) is adequate in the $E$ and $F$ regions of the ionosphere above the geomagnetic equator. This leads to the Cowling conductance $\Sigma_{C}$ and then to $\sigma_{d}$. Nevertheless, only test calculations with different choice of its upper boundary can show the error.

The total current (Eq. 65) in the strip $h_{1}<h<h_{2}$ varies along the equator. If we cut a part from $m$ till $m+\delta m$, the charge conservation law (Eq. 55) for this part takes the shape

$I(m+\delta m)-I(m)+\int_{m}^{m+\delta m} J_{h}\left(m, h_{2}\right) d m=0$.

Let us divide this equation by $\delta m$ and go to the limit $\delta m \rightarrow 0$, then

$\frac{\partial I(m)}{\partial m}+J_{h}\left(m, h_{2}\right)=0$.

Using the denotation of Eq. (65), we obtain

$$
\frac{\partial}{\partial m}\left(A(m) E_{m}(m)\right)+J_{h}\left(m, h_{2}\right)=0 .
$$

This is the boundary condition that must be used at the new boundary $h=h_{2}$ which excludes the boundary layer from the global problem. The second order boundary layer approximation (67) improves the first order approximation (58). Approximations of the next orders are not really used.

When the solution of the 2-D problem is obtained, the electric field at the new boundary $E_{m}(m)$ can be used to calculate inside the boundary layer the electric field and current by Eqs. $(60,63,58,64)$. Also the 3-D current density may be calculated by Ohm's law (24), where $x$ is along the equator as it is $m$. So, the horizontal current density in the equatorial electrojet equals

$$
\begin{aligned}
j_{x} & =\sigma_{P} E_{m}(m)-\sigma_{H} E_{h}(m, h)= \\
& =\left(\sigma_{P}+\sigma_{H} \Sigma_{H} / \Sigma_{P}\right) E_{m}(m) .
\end{aligned}
$$

The coefficient in the last expression

$\sigma_{d}=\sigma_{P}+\sigma_{H} \Sigma_{H} / \Sigma_{P}$

is equal to the local Cowling conductivity $\sigma_{C}=\sigma_{P}+\sigma_{H}^{2} / \sigma_{P}$, if the ionosphere is a homogeneous layer or $\sigma_{H} / \sigma_{P}=$ const at each magnetic field line. Then Eq. (32) gives $\Sigma_{H} / \Sigma_{P}=\sigma_{H} / \sigma_{P}$. The real space distribution of $\sigma_{P}, \sigma_{H}$ do not satisfy any of these conditions. The difference between $\sigma_{d}$ and $\sigma_{C}$ will be demonstrated in Sect. 8 . 

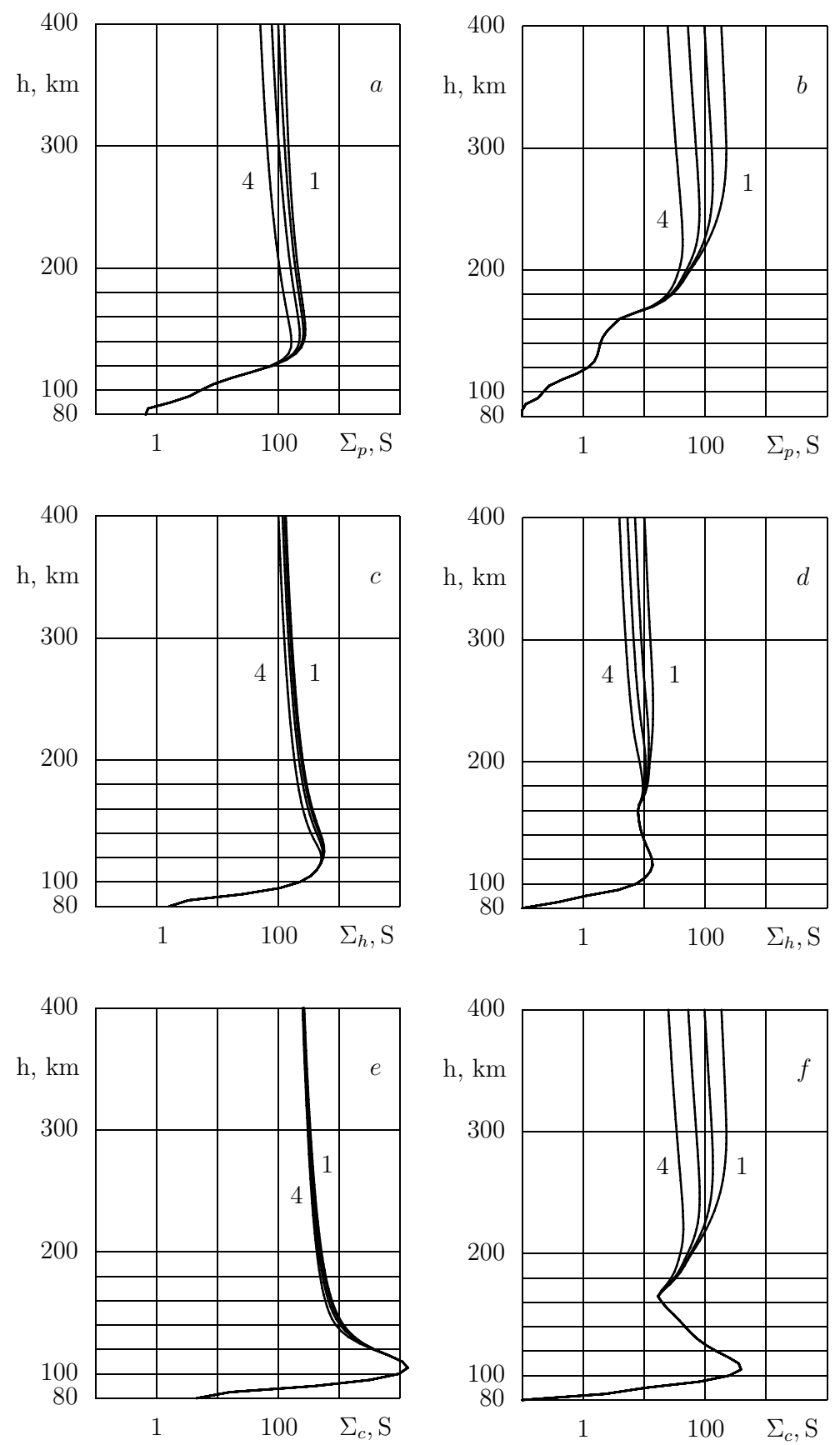

Fig. 5. Conductances $\Sigma_{P}, \Sigma_{H}, \Sigma_{C}$ above the geomagnetic equator (curves 1). The left and right columns present midday and midnight values, respectively. Curves $2,3,4$ are for effective conductances for $t_{0}=1 / 3,1,3 \mathrm{~h}$.

Integration of this parameter $\sigma_{d}$ along a magnetic field line is simple since $\Sigma_{P}, \Sigma_{H}$ are constants for this line

$$
\begin{aligned}
\int \sigma_{d} d l & =\int \sigma_{P} d l+\left(\Sigma_{H} / \Sigma_{P}\right) \int \sigma_{H} d l= \\
& =\Sigma_{P}+\Sigma_{H}^{2} / \Sigma_{P}=\Sigma_{C} .
\end{aligned}
$$

So, the Cowling conductance $\Sigma_{C}$ is not equal to the integral of the local Cowling conductivity as it is sometimes done instead of using the Pedersen and Hall conductances and Eq. (53).

\section{Modification of the ionospheric conductance}

In Sect. 5 , the conductances $\Sigma_{P}, \Sigma_{H}$ are defined for a magnetic field line. We identify a field line by the height $h$ of its position above some point with given geomagnetic 


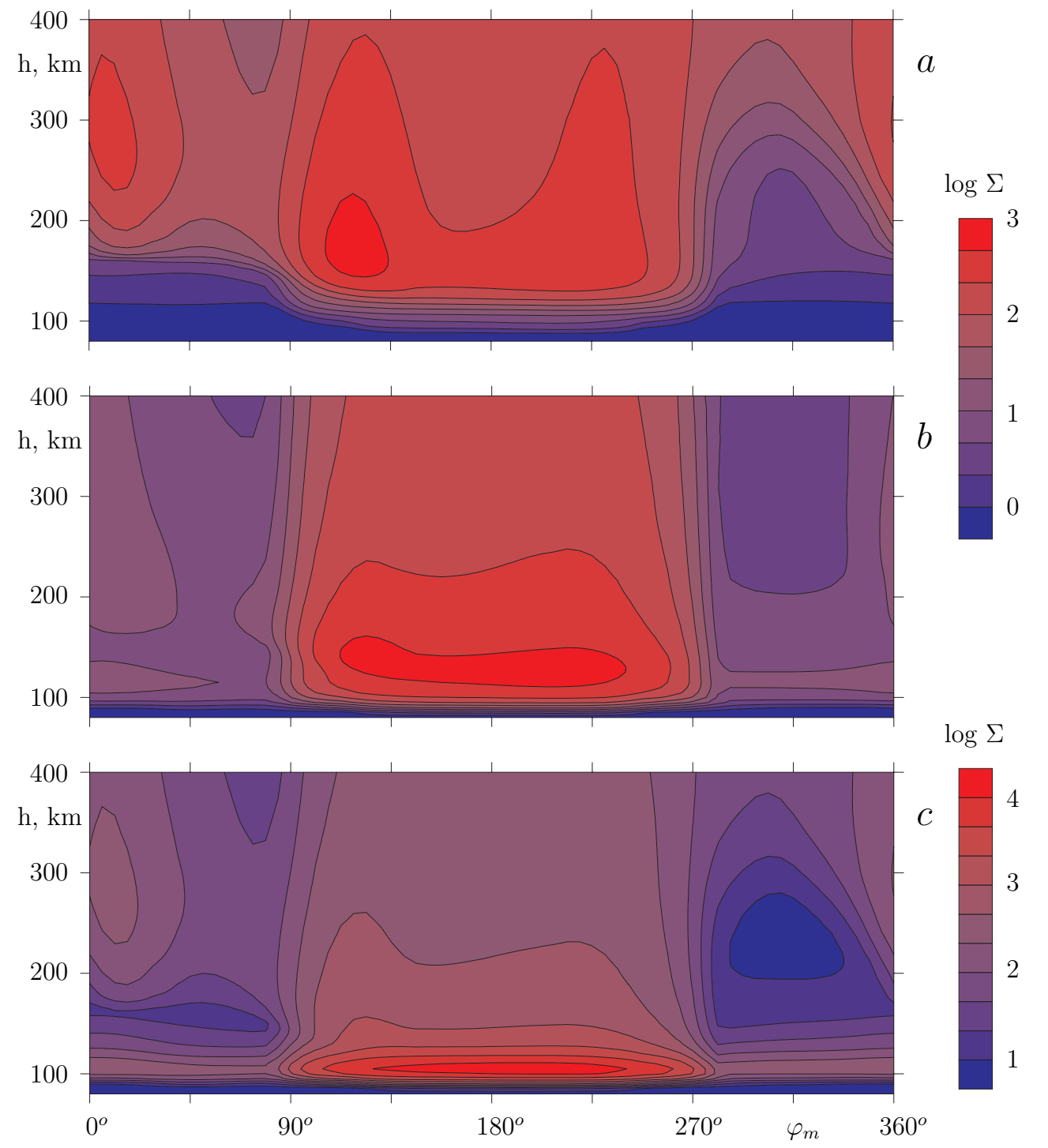

Fig. 6. Conductance distributions at the base surface near the geomagnetic equator for a short-term process. Panel (a) is for $\log _{10}\left(\Sigma_{P}\right)$, panel (b) is for $\log _{10}\left(\Sigma_{H}\right)$, panel (c) is for $\log _{10}\left(\Sigma_{C}\right)$, where conductances in $S$ units are used. The abscissa is the geomagnetic longitude $\varphi_{m}$ and the ordinate $z$ is the height at which the magnetic field line crosses the base surface. The top color scale is for $\Sigma_{P}, \Sigma_{H}$. The bottom color scale is for $\Sigma_{C}$. The values at the neighbor plotted contours differ $\sqrt[3]{10} \simeq 2$ times. The contours $\Sigma_{P}<1 S, \Sigma_{H}<1 S$ and $\Sigma_{C}<10 S$ are not plotted and a common color is used for these small values of $\Sigma$.

coordinates. The conductances $\Sigma_{P}, \Sigma_{H}$ as functions of $h$ are shown in Fig. 5a, b and 5c, d, respectively. The left column presents midday values above the point with geomagnetic coordinates $\theta_{m}=90^{\circ}, \varphi_{m}=0^{\circ}$. The right column presents midnight that is at the point with geomagnetic coordinates $\theta_{m}=90^{\circ}, \varphi_{m}=180^{\circ}$ at a taken moment of universal time.

Figure 5e, $\mathrm{f}$ present the midday and midnight Cowling conductance $\Sigma_{C}$, as it is defined in Sect. 6 .

The curves 1-4 in Fig. 5 have the same meaning as those in Fig. 2. The curves marked with number 1 present the original $\Sigma_{P}, \Sigma_{H}, \Sigma_{C}$ and the curves $2,3,4$ correspond to the processes with $t_{0}=1 / 3,1,3 \mathrm{~h}$. As it is seen in Fig. 5a, c, e, the main modification in the day-side ionosphere is the $\Sigma_{P}$ modification. For $t_{0}=3 \mathrm{~h}$, quantity $\Sigma_{P}$ decreases twice as compared to a short-term process. All three conductances decrease significantly in the night-side ionosphere. The effect under consideration, in fact, cancels the second conducting layer, the conductance of which for the short-term processes is larger than the conductance of the layer below $160 \mathrm{~km}$. Since the Pedersen conductance below $160 \mathrm{~km}$ is small, its 30 times decrease above $200 \mathrm{~km}$ is very important.

Figure 6 shows quantities $\Sigma_{P}, \Sigma_{H}, \Sigma_{C}$ for a short-term process. Conductance distributions at the base surface near 

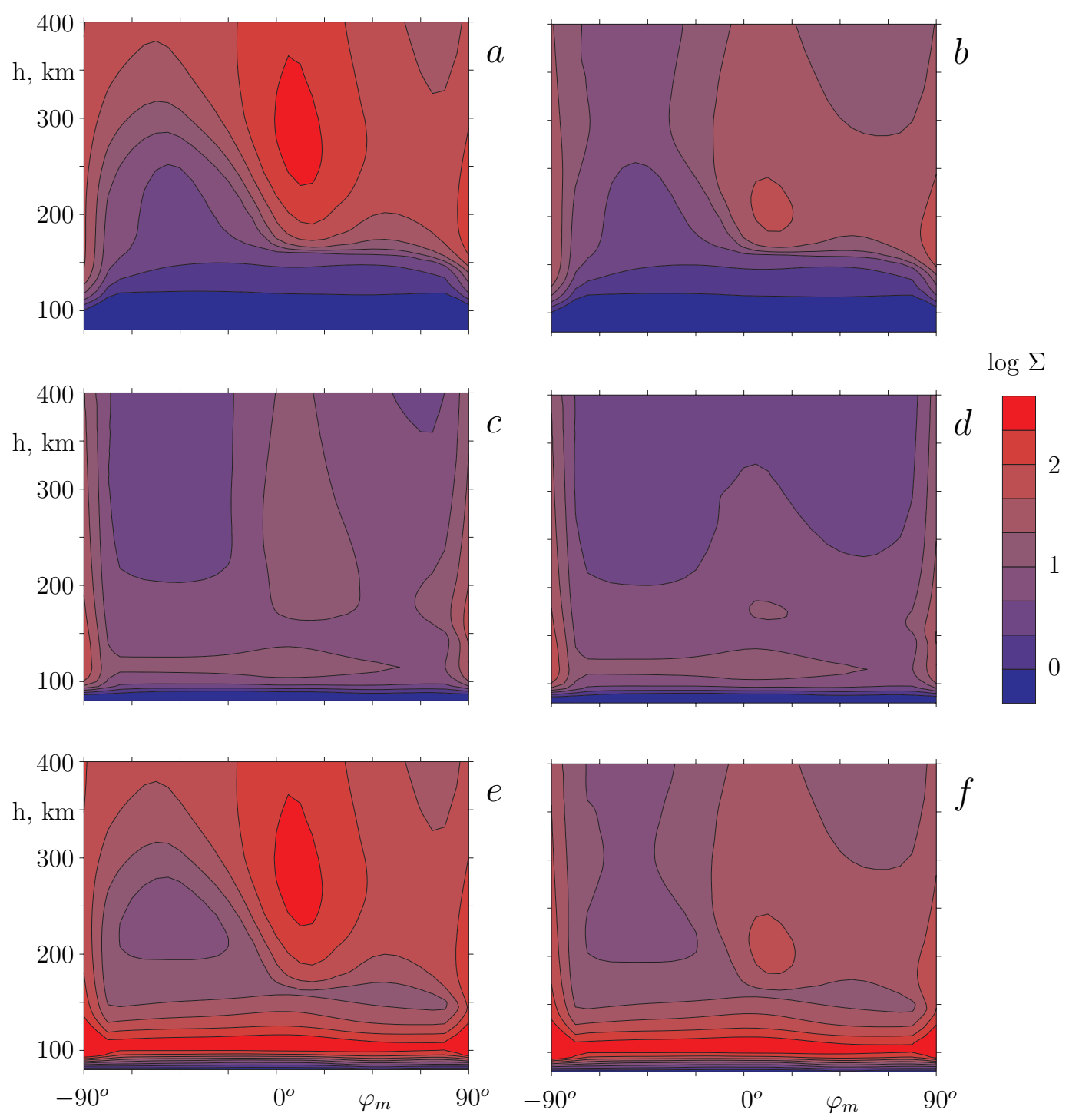

Fig. 7. Conductance distributions above the night half of the geomagnetic equator. Left panels show conductances for a short-term process. Right panels show conductances for a long-term process, for which modifications of the conductivities are done for $t_{0}=3 \mathrm{~h}$. Panels (a), (b) are for $\log _{10}\left(\Sigma_{P}\right)$, panels (c), (d) are for $\log _{10}\left(\Sigma_{H}\right)$, panels (e), (f) are for $\log _{10}\left(\Sigma_{C}\right)$, where conductances in $S$ units are used. The color scale is common for all panels. A common color is used for $\Sigma<1 S$ and the contours $\Sigma<1 S$ are not plotted.

the geomagnetic equator are presented. The abscissa is the geomagnetic longitude $\varphi_{m}$ and the ordinate is the height at which the magnetic field line crosses the base surface, that is shown in Fig. 4. A few magnetic field lines which cross the base surface at the height of $400 \mathrm{~km}$ are plotted in Fig. 4. They correspond to the proper points at the upper boundary $400 \mathrm{~km}$ in every panel of Fig. 6. At the contours plotted in Fig. 6, the conductances have constant values. We use a logarithmic scale for the conductances in units of Siemens. The values of $\Sigma$ to neighboring contours differ $\sqrt[3]{10}$ times which is approximately twice. The color scales as well as values at the contours are shown in the right panel. A special color scale is used for $\Sigma_{C}$ since it has maximal values higher than
$\Sigma_{P}, \Sigma_{H}$. For the purpose to present the main features of the $\Sigma$ distributions, detailed color and contour scales are used. They do not permit to present adequately the sharp decrease of the conductances below $h=100 \mathrm{~km}$. So, the color is not varied there as well as some contours are omitted.

Figure 7 shows the difference between night conductances for short-term and long-term processes by the same meanings which are used in Fig. 6. The left panels Fig. 7a, c, e are the fragments of Fig. 6a, b, c, correspondingly, but centered near midnight. A common color scale with more contrast is used. The right panels of Fig. $7 \mathrm{~b}, \mathrm{~d}$, f present $\Sigma_{P}, \Sigma_{H}, \Sigma_{C}$ for long-term process of $t_{0}=3 \mathrm{~h}$. 
Figure 7 shows that the conductances decrease one order of magnitude above $180 \mathrm{~km}$ in the whole night-side lowlatitude ionosphere for $3 \mathrm{~h}$ long processes as compared to the short-term ones. This is also shown for the midnight in Fig. 5.

The main result of the analyzed acceleration is that for the long-term processes, the night-side height distributions of the conductances become similar to the day-side ones with 30 times less scale, as it takes place in the middle-latitude ionosphere. This is precisely the property which is used practically in all models of the low-latitude ionospheric conductance. Figures 5-7 show that this is wrong for the shortterm processes, but indeed, the analyzed acceleration of the medium permits to ignore the conductance of the higher layer for the long-term processes.

\section{On the night-side equatorial electrojets}

The distributions of the Cowling conductance which are presented in Figs. $6 \mathrm{c}$ and $7 \mathrm{e}$, f permit to analyze possible structure of low-latitude electric current systems.

The coefficients of the two-dimensional conductivity equation in coordinates $h$ and the arc length along the base surface are close to $\Sigma_{P}, \Sigma_{H}$ as it is show above in Sect. 5 . By comparison with these preferential coordinates, a very stretched scale is used in Figs. 6, 7, because the height interval of $320 \mathrm{~km}$ is much less than the length of the equator. Therefore, the presented region of high conductance is to be identified as a narrow strip along the boundary of the conducting domain. The line $h_{1}=80 \mathrm{~km}$ may be taken as an impenetrable boundary.

The total current in the region that includes the magnetic field lines with tops are at the heights $h, h_{1}<h<h_{2} I(m)$ can be calculated as the integral (65).

If we neglect the difference between the base surface and $\theta=90^{\circ}$ plane, then the coordinate $m$ is proportional to $\varphi_{m}$ and $I(m)$ is the current along the equator produced by the electric field in the same direction.

As it is seen in Fig. 6c, quantity $\Sigma_{C}$ has a sharp maximum at $100-110 \mathrm{~km}$ and is large in the height interval $90 \mathrm{~km}$ $<h<130 \mathrm{~km}$. Therefore, the electric current is large just in this region above the equator. It is identified as the day-side equatorial electrojet. An additional view of the electrojet in the plane which is normal to this $m, h$-plane is presented in the end of the section.

The night-side structure is more complicated: One more layer above $200 \mathrm{~km}$ is added. The contribution of the Fregion to the nighttime current at the equator is known (Takeda and Maeda, 1980). The more detailed scale in Fig. 7f shows that its integral contribution is comparable with the contribution of the layer below $120 \mathrm{~km}$ because it is a few times wider. This situation holds for long-term process. For a short-term process, the contribution of this higher layer is one order of magnitude larger than the contribution of the layer below $150 \mathrm{~km}$ as it can be seen in Fig. 7e. The $h$ coordinate in these pictures is the height at which the magnetic field line crosses the geomagnetic equator and it is the maximum height of the line.

The conductances presented in Figs. 6, 7 describe the fictitious conducting film that is defined in Sect. 5. Such a model permits to calculate the electric field $\left(E_{m}, E_{h}\right)$ as the solution of the boundary value problem for Eq. (47). The Ohm law (Eq. 29) gives currents at the fictitious film and real space distributed currents are given by the Ohm law (Eq. 24) with the expression (23) for the electric field in the point of interest.

In the analyzed region $80 \mathrm{~km}<h<400 \mathrm{~km}$ near the equator, the Eqs. (60) and (23) mean

$$
\left(\begin{array}{c}
E_{x} \\
E_{y}
\end{array}\right)=\left(\begin{array}{c}
1 \\
-\frac{\Delta x_{2}}{\Delta y_{2}}-\frac{\Delta x_{1}}{\Delta y_{2}} \frac{\Sigma_{h m}}{\Sigma_{h h}}
\end{array}\right) \frac{g \Delta m}{\Delta x_{1}} E_{m}(m) .
$$

The Ohm law (Eq. 24) gives a current which has both, horizontal and vertical components, in contrast to the integral current, because the local vertical current at one part of the magnetic field line can be compensated by negative vertical current at another part of the line, so that the total current at the line has a zero vertical component $J_{h}=0$.

If we neglect geometrical factors what means no difference between $d x, d y$ and $g d m, d h$ when the simplified expression (33) is valid, then Eqs. (72) and (24) permit to calculate the current density as

$$
\left(\begin{array}{l}
j_{x} \\
j_{y}
\end{array}\right)=\left(\begin{array}{cc}
\sigma_{P} & -\sigma_{H} \\
\sigma_{H} & \sigma_{P}
\end{array}\right)\left(\begin{array}{c}
1 \\
-\Sigma_{H} / \Sigma_{P}
\end{array}\right) E_{x} .
$$

So, the horizontal component of the current density is

$j_{x}=\sigma_{d} E_{x}$,

and the parameter $\sigma_{d}$ is defined by Eq. (70).

It should be mentioned that the remaining z-component of the current density that is field-aligned in this case, can be calculated from the charge conservation law (Eq. 38) after $j_{x}, j_{y}$ are calculated by Eq. (73).

The difference between $\sigma_{d}$ and the local Cowling conductivity $\sigma_{C}$ is discussed in Sect. 6 . The difference between their values can be seen by comparing Fig. $8 \mathrm{a}$ and $\mathrm{c}$ for the night time ionosphere, and Fig. 9a and $\mathrm{c}$ which present the day time cross-sections of the equatorial ionosphere.

The Cowling conductivity has a maximum value near $h=100 \mathrm{~km}$, because of that, quantity $\sigma_{H}$ has maximum values at the heights $100-120 \mathrm{~km}$ and $\sigma_{P}$ is much less there, as it can be seen in Fig. 2. If the whole magnetic field line is situated below $120 \mathrm{~km}$, then the ratio of the integral conductances $\Sigma_{H} / \Sigma_{P}$ is of the same order of magnitude as the maximal value of $\sigma_{H} / \sigma_{P}$. Then $\sigma_{d}$ does not differ much from $\sigma_{C}$ in the points of these lines, which occupy the region $\pm 3^{\circ}$ near the magnetic equator. If a part of the magnetic field line is above $120 \mathrm{~km}$ then the integral conductance $\Sigma_{P}$ increases much in contrast to $\Sigma_{H}$, since $\sigma_{P}$ is much larger and $\sigma_{H}$ is much smaller there, as compared to their values below 


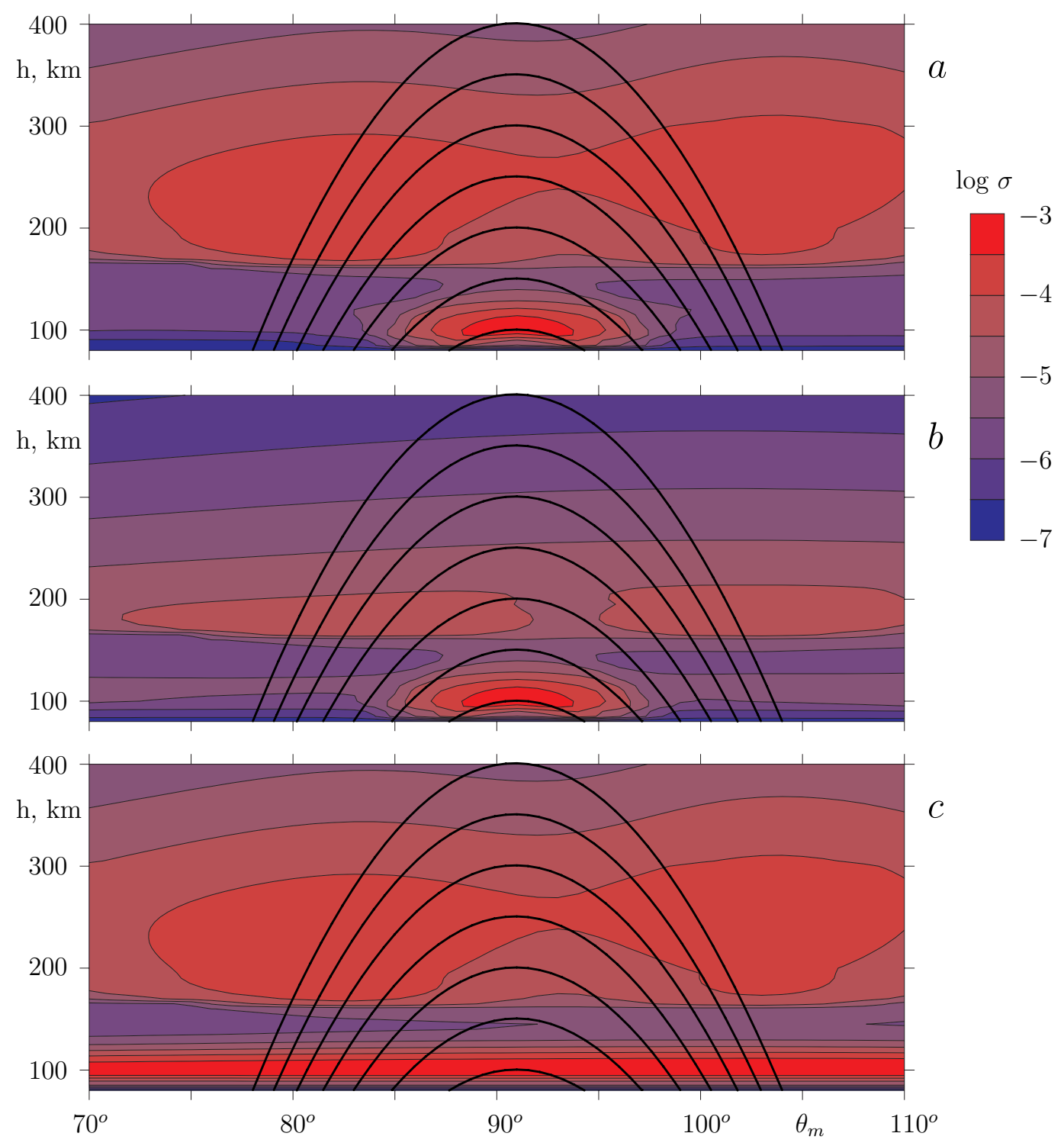

Fig. 8. Conductivity distributions at the surface that consists of the magnetic field lines which cross the geomagnetic equator above the point $\varphi_{m}=0$. There is midnight in this point. A few magnetic field lines of this sort are plotted. Panel (a) is for $\log _{10}\left(\sigma_{d}\right)$ for a short-term process, panel (b) is for $\log _{10}\left(\sigma_{d}\right)$ for a long-term process, panel (c) is for $\log _{10}\left(\sigma_{C}\right)$ for a short-term process, where conductivities in $S / m$ units are used. The abscissa is the geomagnetic colatitude $\theta_{m}$ and the ordinate $h$ is the height. The color scale is common for all panels. The values at the neighbor plotted contours differ $\sqrt{10} \simeq 3.2$ times.

$120 \mathrm{~km}$. Therefore, $\Sigma_{H} / \Sigma_{P}$ is much less than the maximal value of $\sigma_{H} / \sigma_{P}$ and $\max \sigma_{d} \ll \max \sigma_{C}$ at such a magnetic field line. This dramatic difference between $\sigma_{d}$ and $\sigma_{C}$ would not exist if $\sigma_{P}, \sigma_{H}$ would have common height distributions. The upper boundary of the region, where $\sigma_{d}$ can be used is defined by the same restrictions as those for $\Sigma_{C}$, which are mentioned in Sect. 6.

When the local Cowling conductivity $\sigma_{C}$ is used, the limitations are stated as "several degrees away from the magnetic equator". This statement is rather indefinite and a comparison of Fig. 9a with $\mathrm{c}$ shows that the limitation is very sharp.
It is well seen in Fig. 9c that it is hard to cut a proper part from the layer in that $\sigma_{C}$ is almost homogeneous in the direction across the equator. In contrast, we see just the region of the electrojet in the $\sigma_{d}$ distributions shown in Fig. 9a and $9 \mathrm{~b}$. This region is a sequence of large values of the Cowling conductance $\Sigma_{C}$ for this group of magnetic field lines.

The integrals

$\int j_{x} d h, \quad \int j_{z} d h$

define some two-dimensional current density. If the horizontal scale is much larger than $\Delta h$, this current may be put at 


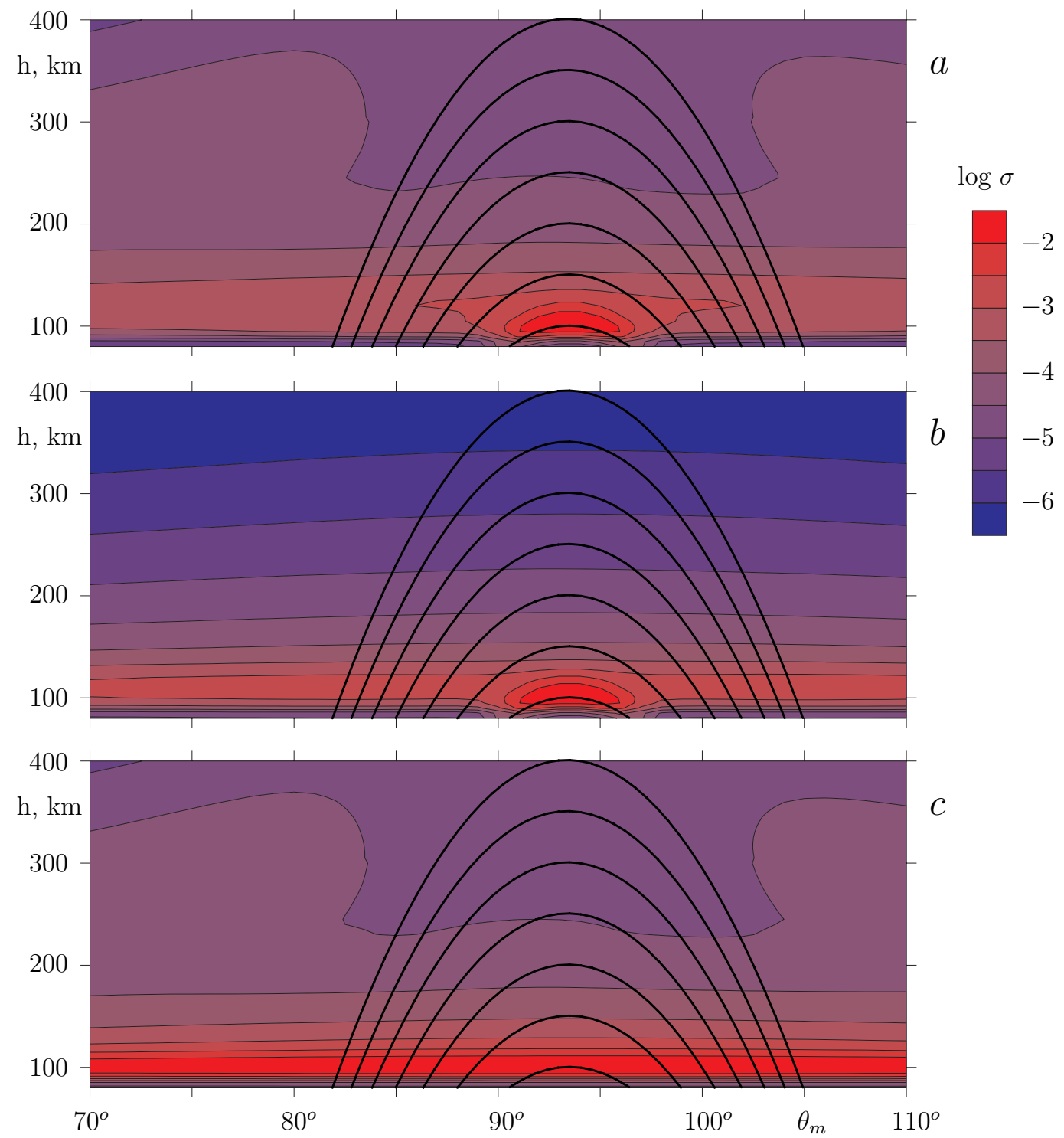

Fig. 9. The same as Fig. 8 but near the midday point $\varphi_{m}=180^{\circ}$ at the geomagnetic equator.

the sphere $h=100 \mathrm{~km}$ and used for an approximate calculation of the magnetic perturbations below the ionosphere instead of being distributed in space current $\mathbf{j}$. So, the vector function (75) is referred to as the equivalent current density.

Since the $\mathrm{x}$-direction is normal to the plane of Fig. 8 and approximately $E_{x}=$ const in the domain presented in Fig. 8, these maps also present the current density (74) in the vicinity of the geomagnetic equator in $\mathrm{A} / \mathrm{m}$ units for $E_{x}=1 \mathrm{~V} / \mathrm{m}$. Figure 9 may be used in the same manner for midday.

Figure 9a shows the concentration of the current in the jet with a horizontal size of about $600 \mathrm{~km}$. This panel is plotted for a short-term process when the ionospheric plasma has zero velocity and the next panel presents the results of plasma acceleration. Figure $9 \mathrm{~b}$ demonstrates that almost the same jet appears during long-term processes with $\tau=3 \mathrm{~h}$, but the modification of the conductivity is valuable outside of the jet.

The conductivity in the F-region is much more important in the nighttime ionosphere. So, the effect of the modification of the conductivity is stronger. This is demonstrated in Fig. 8a, b. There is a region of a jet and it has about the same size as in the daytime, but there is one more conductor in the F-region that is switched in parallel to the jet region, since they have the same $E_{x}=$ const. For a short term process, the parameters of these two conductors are more obvious in Fig. $7 \mathrm{e}$ since the line $\varphi_{m}=0$ presents just integrals along the magnetic field lines of the conductivity $\sigma_{d}\left(\theta_{m}, h\right)$ as presented in Fig. 8a.

The same relation for a long-term process exists between Figs. $7 \mathrm{f}$ and Fig. $8 \mathrm{~b}$. We see that the conductor above $150 \mathrm{~km}$ 
has an about ten times larger integral conductance. So, only about one tenth of the current is below $150 \mathrm{~km}$. It is concentrated as the equatorial jet in the nighttime ionosphere. The main part of the current occupies the horizontal strip near the equator of about $4000 \mathrm{~km}$ width, as it is seen in Fig. 8a. Since this conductor has minimal conductance just above the jet region, the jet hardly can be identified from ground-based measurements of magnetic perturbations.

The conductivity $\sigma_{d}$ for a long-term process with $\tau=3 \mathrm{~h}$ is presented in Fig. 8b. The region of the jet is almost the same as for a short-term process in Fig. 8a, but the second conductor is by about twenty times reduced, what is better seen in Fig. 7f. So, the jet becomes the main conductor and it may be identified by ground based measurements if the total current along the nighttime part of the equatorial ionosphere is strong enough.

It is also important to note that the conductances in the night-side low-latitude ionosphere are much larger than it can be expected if one extrapolates their properties from the middle-latitudes to low latitudes.

We can summarize the results of this section as the expectation of essential modifications of the model electric fields and currents in the low-latitude ionosphere compared to the models which use less detailed conductivity models than IRI gives. Our models (Denisenko and Zamay, 1992) are among the latter.

For the long-term processes the modification of the traditional models may be moderate because on one hand the IRI2001 model gives the second night-side layer of high conductance, and on the other hand, the acceleration of the medium reduces the current in such a layer.

\section{Discussion}

The reduction of conductivities as obtained in this paper is of the same order as the one in the model by Akasofu and Dewitt (1965) which represents the modification of the Pedersen conductivity for an electric field being harmonic in time $(\exp (i \omega t))$ in the middle-latitude day time ionosphere. The effective conductivity does not differ much from the steady state conductivity for processes whose duration is much less than one hour. If the time scale of a process is of about few hours, then the conductivity above $200 \mathrm{~km}$ decreases by one order of magnitude.

The difference between the conductivities $\sigma_{P}, \sigma_{H}$ and their effective values $\left\langle\sigma_{P}\right\rangle,\left\langle\sigma_{H}\right\rangle$ (Eqs. 8, 9) in the Earth's ionosphere may be qualified as the reduction or even exclusion of conductance in the $F_{2}$ layer for $h>200 \mathrm{~km}$ for longterm processes.

In addition to the results by Akasofu and Dewitt (1965), we analyzed in detail this effect in the nighttime and lowlatitude parts of the ionosphere. It is shown that the reduction of conductivity is more important in the nighttime iono- sphere, since $\sigma_{P}$ is large in the $F$ layer in contrast to the day time ionosphere.

Also, we have calculated the modified values of the conductances $\left\langle\Sigma_{P}\right\rangle,\left\langle\Sigma_{H}\right\rangle,\left\langle\Sigma_{C}\right\rangle$ which are necessary for models of large-scale electric fields and currents while the research (Akasofu and Dewitt, 1965) was concentrated on the modification of the local conductivities $\sigma_{P}, \sigma_{H}, \sigma_{\|}$and their role for the electric field penetration from the ionosphere into the magnetosphere.

We have shown the modification of the conductivities only in some typical conditions in this paper. Real parameters of the ionosphere may be very different. For example, the nighttime F-region has a large variation with solar activity. The midnight conductances $\Sigma_{P}, \Sigma_{H}$ and $\Sigma_{C}$ at the magnetic field lines, which tops are in the F-region, are presented in Fig. 5b, d, f. They are calculated for a moderate value of Covington Index 130 in this paper. These $\Sigma_{P}, \Sigma_{H}$ and $\Sigma_{C}$ in the F-region can be four times less or twice larger, when Covington Index equals 70 or 190.

Real processes have different variations in time. For a periodical process, the approach (Akasofu and Dewitt, 1965) is better since it is based on functions harmonic in time. If we are interested in some switching from one steady state to another, our approach is more adequate since it describes just a relaxation. One of the advantages of our model for more complicated processes is that $\left\langle\sigma_{P}\right\rangle,\left\langle\sigma_{H}\right\rangle$ are real numbers in contrast to complex values in the model from (Akasofu and Dewitt, 1965). The imaginary parts of that values represent the time delay in the conductivity modification. The parameter $\tau$ (Eq. 6) represents the delay in our approach.

The proposed modification of the local conductivity according to Eqs. $(8,9)$ as well as the model (Akasofu and Dewitt, 1965) is based on the assumption (4) that no force but the Ampére one accelerates the medium. The Ampére force above the geomagnetic equator may be vertical as well as horizontal. The vertical movement of the ionospheric medium can break pressure balance stronger than the horizontal movement. In spite of that, such a motion due to an Eastern or Western electric field is often observed above the geomagnetic equator as the quiet time fountain effect or super-fountain effect during storm times (Tsurutani et al., 2004).

Pressure and friction are supposed to be unchanged in our model. Conversely, the original local conductivities $\sigma_{P}, \sigma_{H}$ remain unchanged if the Ampere force is negligible. The adequateness of one of these opposite assumptions can be proved only in the frame of a more general model, because of the fact that pressure and other parameters may be changed as a result of the motion. A pressure gradient was taken into account in the frame of a local model by Maeda (1977).

We mention, that it would be more precise not to modify the conductivity but to follow the plasma motion. This results, however, a much more complicated model, since it becomes time dependent. 
If we have only the simplified model of the ionosphere that is a global conductor with given conductivity and velocity distributions, it is useful to calculate the electric fields and currents twice, for given $\sigma_{P}, \sigma_{H}$ and for effective $\left\langle\sigma_{P}\right\rangle$, $\left.<\sigma_{H}\right\rangle$. If the results are different, a qualitative analysis of acceleration is needed. In special cases, this examination supports one of the alternatives. For example, a considerable reduction of the effective conductivity in the $F_{2}$ layer must be always done. In general, a quantitative simulation of the motion is necessary.

Additional questions appear when the model of the ionospheric conductor (47) is used in the dynamo theory. If the space distribution of the velocity is known, it is possible to calculate the extrinsic current (Eq. 40), its divergence $q$ (Eq. 39), and the proper term $Q$ (Eq. 45) on the righthand side of Eq. (47). The modification of the conductivity $($ Eqs. 8,9$)$ can not be done in such a case, since the velocity is given. A small time of acceleration in the upper ionosphere that is usually referred to as the motor region means that the velocity of the medium is defined not by the neutral winds but mainly by the electric fields there. It ought be taken into account in the definition of the velocity that may be taken as given only in the dynamo region, where the time of the medium acceleration is large enough. It also should be mentioned that the components of the velocity not horizontal but normal to the magnetic field are of value.

\section{Conclusions}

The IRI model gives the second high conductance layer in the night-side low-latitude ionosphere in addition to the main conductor in the E-layer. It reduces the electric field and equatorial electrojets, but intensifies the night-side currents in the $F_{2}$ layer during short-term events. These currents occupy the regions which are much wider than those of the equatorial electrojets.

The local conductivity is to be reduced with Eqs. $(8,9)$ to take into account the influence of the Ampére force. The corresponding acceleration of the conducting medium reduces the currents in the $F_{2}$ layer. This reduction is maximal in the low-latitude night-side ionosphere.

The presented theory predicts different space distributions of the currents in the night time low-latitude ionosphere during long-term and short-term events. The long-term currents are expected to be in the E-layer and are concentrated in the electrojet of about the same width as the daytime jet width. During the short-term events the nighttime jet is masked by a much wider current in the F-layer. The electric field above the geomagnetic equator produced by the same voltage in high latitudes is expected to be much less during the short-term events since the total conductance of the equatorial ionosphere decreases much because of the medium acceleration. This behavior is important for the analysis of the electric field penetration from the auroral zone till the geomagnetic equator.

It is demonstrated on the basis of the IRI-2001 model that the parameter (70) $\sigma_{d}=\sigma_{P}+\sigma_{H} \Sigma_{H} / \Sigma_{P}$, that involves the Pedersen and Hall conductances $\Sigma_{P}, \Sigma_{H}$ of the whole magnetic field line, define the space distribution of the electric current density in the equatorial ionosphere and the local Cowling conductivity $\sigma_{C}=\sigma_{P}+\sigma_{H}^{2} / \sigma_{P}$ can not be used for this purpose.

Both, $\sigma_{d}$ and $\sigma_{C}$, appear when a magnetic field line is near a nonconducting domain what means that there is zero current through the boundary of this domain. Then, the proper component of the current across the magnetic field direction is regarded as to be a small one in some vicinity of this boundary.

The main difference between $\sigma_{d}$ and $\sigma_{C}$ is that the $\sigma_{d}$ definition includes the possibility for the electric current to flow along a magnetic field line in order to close all currents $\mathbf{j}_{\perp}$ which go to this line from neighboring ones. The local Cowling conductivity $\sigma_{C}$ corresponds to a $\mathbf{j}_{\perp}$ closure at each point of the magnetic field line, which is adequate only if the fieldaligned conductivity equals zero or the field-aligned current equals zero because of some symmetry. Such a symmetric object is a magnetic field line with constant local conductivity at the whole line and a generator must be also constant if it exists, but there $\sigma_{C}=\sigma_{d}$ in this case. The first possibility of zero field-aligned conductivity is not appropriate for the ionosphere. So, we see no reason to use local Cowling conductivity while the much more adequate parameter $\sigma_{d}$ exists. A similar parameter was introduced long time ago by Gurevich et al. (1976). This was done in a more complicated form for the more general case in nonorthogonal coordinates. It seems that such a complicated form explains why it is not widely used until now.

The problem of the local Cowling conductivity was discussed by Forbes (1981) in the frame of a 2-D model similar to the model of Gurevich et al. (1976) that we use here. In such a model $\Sigma_{C}=\Sigma_{P}+\Sigma_{H}^{2} / \Sigma_{P}$. The so called "thinshell model" which leads to $\sigma_{C}$ was also analyzed by Forbes (1981). He did not at all reject the usage of "thin-shell model" and proper $\sigma_{C}$, and left it to be used with the rather indefinite restriction "several degrees away from the magnetic equator", while, in fact, he did not use such an approximation in his model. Here we have performed the next step, which is natural in such an approach, and have changed $\sigma_{C}$ by more general parameter $\sigma_{d}$.

Acknowledgements. This work is supported by grant 07-05-00135 from the Russian Foundation for Basic Research, by the Programs 16.3 and 2.16 of the Russian Academy of Sciences, and by project I.2/04 from "Österreichischer Austauschdienst". It is also supported by the Austrian "Fonds zur Förderung der wissenschaftlichen Forschung" under projects P17100-N08 and P20145-N16. We acknowledge support by the Austrian Academy of Sciences, "Verwaltungstelle für Auslandsbeziehungen", and the Russian Academy of Sciences. 
Part of this research was done during academic visits of V. V. Denisenko to the Space Research Institute of the Austrian Academy of Sciences in Graz as well as during an academic visit of H. K. Biernat to the Institute of Computational Modelling of the Russian Academy of Sciences in Krasnoyarsk.

We are grateful to the referees for fruitful discussions.

Topical Editor M. Pinnock thanks S. M. Stankov and another anonymous referee for their help in evaluating this paper.

\section{References}

Akasofu, S.-I. and Chapman, S.: Solar-Terrestrial Physics, Clarendon Press, Oxford, 1972.

Akasofu, S.-I. and Dewitt, R. N.: Dynamo action in the ionosphere and motions of the magnetospheric plasma. III. The Pedersen conductivity, generalized to take account of acceleration of the neutral gas, Planet. Space Sci., 13, 737-744, 1965.

Banks, P.: Collision frequencies and energy transfer, electrons, Planet. Space Sci., 14, 1085-1103, 1966.

Danilov, A. D. and Smirnova, N. V.: Improving the 75 to $300 \mathrm{~km}$ ion composition model of the IRI, Adv. Space Res., 15, 171-177, 1995.

Denisenko, V. V.: A boundary value problem for an elliptic equations with asymmetric coefficients in a non-schlicht domain, Siberian Mathematical Journal, 43, 1055-1068, 2002.

Denisenko, V. V. and Zamay, S. S.: Electric field in the equatorial ionosphere, Planet. Space Sci., 40, 941-952, 1992.

Forbes, J. M.: The equatorial electrojet, Rev. Geophys., 19, 469504, 1981.

Gurevich, A. V., Krylov, A. L., and Tsedilina, E. E.: Electric field in the Earth's magnetosphere and ionosphere, Space Sci. Rev., 191, 59-160, 1976.

Hargreaves, J. K.: The upper atmosphere and Solar-Terrestrial relations, Van Nostrand Reinold Co. Ltd, NY, 1979.
Hedin, A. E.: Extension of the MSIS Thermospheric Model into the Middle and Lower Atmosphere, J. Geophys. Res., 96, 1159$1165,1991$.

Hedin, A. E., Salah, J. E., and Evans, J. V.: A global thermospheric model based on mass spectrometer and incoherent scatter data. MSIS. 1. $\mathrm{N}_{2}$ density and temperature. 2. Composition, J. Geophys. Res., 82, 2139-2156, 1977.

Korn, G. A. and Korn, T. M.: Mathematical handbook, McGrawHill Book Cmpany, NY, 1968.

Maeda, K.: Conductivity and drifts in the ionosphere, J. Atmos. Terr. Phys., 39, 1041-1053, 1977.

Models Distribution and Staging Directory, National Space Science Data Center, NASA, ftp:/nssdcftp.gsfc.nasa.gov/models, 2006.

Namgaladze, A. A., Foerster, M., and Yurik, R. Yu.: Analysis of the positive ionospheric response to a moderate geomagnetic storm using a global numerical model, Ann. Geophys., 18, 461-477, 2000 , http://www.ann-geophys.net/18/461/2000/.

Stening, R. J.: Modelling the equatorial electrojet, J. Geophys. Res., 90, 4083-4098, 1985.

Stubbe, P.: Frictional forces and collision frequencies between moving ion and neutral gases, J. Atmos. Terr. Phys., 30, 19651985, 1968.

Takeda, M. and Maeda, H.: Three-dimensional structure of ionospheric currents. 1. Currents caused by diurnal tidal winds, J. Geophys. Res., 85, 6895-6899, 1980.

Tsurutani, B., Mannucci, A., Iijima, B., Abdu, M. A., Sobral, J. H. A., Gonzalez, W., Guarnieri, F., Tsuda, T., Saito, A., Yumoto, K., Fejer, B., Fuller-Rowell, T. J., Kozyra, J., Foster, J. C., Coster, A., and Vasyliunas, V. M.: Global dayside ionospheric uplift and enhancement associated with interplanetary electric fields, J. Geophys. Res., 109, A08302, doi:10.1029/2003JA010342.

Vanyan, L. L., Zeleny, L. M., and Parshev, V. A.: On the frequency dispercion of conductivity of ionospheric plasma, Geomagn. Aeronomy, 13, 188-190, 1973. 